TRANSACTIONS OF THE

AMERICAN MATHEMATICAL SOCIETY

Volume 352, Number 2, Pages 553-577

S 0002-9947(99)02172-8

Article electronically published on May 20, 1999

\title{
FACTORIZATION IN GENERALIZED POWER SERIES
}

\author{
ALESSANDRO BERARDUCCI
}

\begin{abstract}
The field of generalized power series with real coefficients and exponents in an ordered abelian divisible group $\mathbf{G}$ is a classical tool in the study of real closed fields. We prove the existence of irreducible elements in the ring $\mathbf{R}\left(\left(\mathbf{G}^{\leq 0}\right)\right)$ consisting of the generalized power series with non-positive exponents. The following candidate for such an irreducible series was given by Conway (1976): $\sum_{n} t^{-1 / n}+1$. Gonshor (1986) studied the question of the existence of irreducible elements and obtained necessary conditions for a series to be irreducible. We show that Conway's series is indeed irreducible. Our results are based on a new kind of valuation taking ordinal numbers as values. If $\mathbf{G}=(\mathbf{R},+, 0, \leq)$ we can give the following test for irreducibility based only on the order type of the support of the series: if the order type is either $\omega$ or of the form $\omega^{\omega^{\alpha}}$ and the series is not divisible by any monomial, then it is irreducible. To handle the general case we use a suggestion of M.-H. Mourgues, based on an idea of Gonshor, which allows us to reduce to the special case $\mathbf{G}=\mathbf{R}$. In the final part of the paper we study the irreducibility of series with finite support.
\end{abstract}

\section{INTRODUCTION}

1.1. Fields of generalized power series. Generalized power series with exponents in an arbitrary abelian ordered group are a classical tool in the study of valued fields and ordered fields [Hahn 07, MacLane 39, Kaplansky 42, Fuchs 63, Ribenboim 68, Ribenboim 92].

Given a field $K$ and an ordered abelian group $\mathbf{G}=(\mathbf{G},+, 0, \leq)$, the field $K((\mathbf{G}))$ of generalized power series consists of all formal sums $a=\sum_{\gamma} a_{\gamma} t^{\gamma}$ with coefficients $a_{\gamma}$ in $K$, exponents $\gamma \in \mathbf{G}$ and well-ordered support

$$
S_{a}=\left\{\gamma \in \mathbf{G} \mid a_{\gamma} \neq 0\right\}
$$

in the induced order of $\mathbf{G}$. Another notation for $K((\mathbf{G}))$ is $K((t))^{\mathbf{G}}$, where the formal variable $t$ is displayed. We always use $t$ for the formal variable. Addition of two series is defined in the obvious way. The fact that the support is well ordered (i.e. it contains no infinite descending chain) makes it possible to define the multiplication of two series by the usual convolution product: $\left(\sum_{\alpha} a_{\alpha} t^{\alpha}\right)\left(\sum_{\beta} b_{\beta} t^{\beta}\right)=\sum_{\gamma} c_{\gamma} t^{\gamma}$, where $c_{\gamma}=\sum_{\alpha+\beta=\gamma} a_{\alpha} b_{\beta}$. (One must check that only finitely many terms in this

Received by the editors September 12, 1996 and, in revised form, July 22, 1997.

1991 Mathematics Subject Classification. Primary 06F25; Secondary 13A16, 03H15, 03E10, 12J25, $13 \mathrm{~A} 05$.

Key words and phrases. Generalized power series, ordered rings, surreal numbers, open induction, real closed fields, valuations, ordinal numbers.

The results of this paper were presented at the A.S.L. meeting at S. Sebastian, July 9 - 15, 1996, and at the meeting "Model Theory of Fields", Durham, July 22 - Aug. 1, 1996.

(C)1999 American Mathematical Society 
summation are non-zero and that the set of $\gamma$ with $c_{\gamma} \neq 0$ is well ordered.) It can be shown that every non-zero series has an inverse and therefore $K((\mathbf{G}))$ is a field [Hahn 07] (see [Neumann 49] for the case of division rings).

On $K((\mathbf{G}))$ we have a natural valuation:

$$
\begin{array}{r}
v: K((\mathbf{G})) \rightarrow \mathbf{G} \\
a \mapsto \text { least element of } S_{a}
\end{array}
$$

If $K$ is an ordered field, then we can define an order on $K((\mathbf{G}))$ by declaring an element $a \in K((\mathbf{G}))$ positive if its leading coefficient (i.e. the coefficient of $t^{v(a)}$ ) is a positive element of $K$.

It is a classical result that if $K$ is real closed and $\mathbf{G}$ is divisible then $K((\mathbf{G}))$ is also real closed. Moreover any ordered field, hence any real closed field $F$, admits a power series representation with real coefficients [Gleyzal 37]. More precisely, $F$ can be embedded as an ordered field in $\mathbf{R}((\mathbf{G}))$, where $\mathbf{R}$ is the ordered field of real numbers and $\mathbf{G}$ is the group of archimedean classes of $F$. Indeed, [Krull 32] shows that $F$ possesses a "maximal" extension, and [Kaplansky 42, Theorem 6, Theorem 8] proves that the maximal extension is necessarily a power series field.

1.2. Integer parts of real closed fields. An integer part of an ordered field $F$ (usually assumed to be real closed) is an ordered subring $Z$ having 1 as its least positive element and such that for each $a \in F$ there is $b \in Z$ (necessarily unique) such that $b \leq a<b+1$. Using generalized power series, [Mourgues-Ressayre 93] proved that every real closed field $F$ has an integer part. [Boughattas 93] showed that real closeness is necessary. If $F$ is a subfield of the reals $\mathbf{R}$ then its only integer part is the ring of integers $\mathbf{Z}$. However if $F$ is non-archimedean it can have non-isomorphic integer parts, or even not elementarily equivalent ones (each one contains an isomorphic copy of the integers $\mathbf{Z}$ as a convex subring). Ressayre has exploited integer parts to give a new proof of Wilkie's theorem on the model completeness of the reals with exponentiation, and to give a complete axiomatization of the elementary properties of the exponential function [Ressayre 93, Ressayre 95]. (See [Dries et al. 94] for a related proof not using integer parts.)

Integer parts of real closed fields happen to coincide with the models of the axiom system known as open induction [Shepherdson 64], and a lot of work has been done to study the properties of these discrete rings, focusing in particular on the solution of diophantine equations (see [Wilkie 78, Dries 80-1, Dries 80-2, Otero 90, Otero 93-1]) and on the behavior of primes [Macintyre-Marker 89, Berarducci - Otero 96, Moniri 94, Biljacovic 96]. Generalized power series were exploited in [Otero 93-2] to prove the joint embedding property for normal models of open induction.

1.3. A problem of Conway and Gonshor: existence of irreducible elements in the ring $\mathbf{R}\left(\left(\mathbf{G}^{\leq 0}\right)\right)$. Let us specialize the notion of integer part to the ordered field $\mathbf{R}((\mathbf{G})$ ), where $\mathbf{G}$ is a divisible ordered abelian group (hence a $\mathbf{Q}$ vector space). In this case it is easy to extract an integer part using the direct sum decomposition:

$$
\mathbf{R}((\mathbf{G}))=\mathbf{R}\left(\left(\mathbf{G}^{<0}\right)\right) \oplus \mathbf{R} \oplus \mathbf{R}\left(\left(\mathbf{G}^{>0}\right)\right)
$$

where $\mathbf{R}\left(\left(\mathbf{G}^{<0}\right)\right)$ consists of the series with negative exponents and $\mathbf{R}\left(\left(\mathbf{G}^{>0}\right)\right)$ consists of those with positive exponents. We have:

$$
\mathbf{R}\left(\left(\mathbf{G}^{<0}\right)\right) \oplus \mathbf{Z} \text { is an integer part of } \mathbf{R}((\mathbf{G}))
$$


Any integer part is a discrete ring, so in particular it contains no infinitesimal elements. The set of all infinitesimal elements of $\mathbf{R}((\mathbf{G}))$ is easily seen to be $\mathbf{R}\left(\left(\mathbf{G}^{>0}\right)\right)$, while $\mathbf{R}\left(\left(\mathbf{G}^{>0}\right)\right) \oplus \mathbf{R}$ is the set of all finite elements. All the elements of $\mathbf{R}\left(\left(\mathbf{G}^{<0}\right)\right)$ are infinite.

The ring $\mathbf{R}\left(\left(\mathbf{G}^{<0}\right)\right) \oplus \mathbf{Z}$ is not the only integer part of $\mathbf{R}((\mathbf{G}))$, but it is the only one which is truncation closed, namely it has the property that if a series $\sum_{\beta} b_{\beta} t^{\beta}$ belongs to it, then also its truncations $\sum_{\beta<\alpha} b_{\beta} t^{\beta}$ belong to it. Truncation closedness plays a crucial role in the work of Ressayre and his collaborators.

The primes of $\mathbf{Z}$ remain prime in $\mathbf{R}\left(\left(\mathbf{G}^{<0}\right)\right) \oplus \mathbf{Z}$, and it is a natural question whether $\mathbf{R}\left(\left(\mathbf{G}^{<0}\right)\right) \oplus \mathbf{Z}$ contains irreducible elements not in $\mathbf{Z}$. This is easily seen to be equivalent to the question of whether the ring

$$
\mathbf{R}\left(\left(\mathbf{G}^{\leq 0}\right)\right):=\mathbf{R}\left(\left(\mathbf{G}^{<0}\right)\right) \oplus \mathbf{R}
$$

has any irreducible element, and we will consider the problem in this latter form. Since this ring is not noetherian the answer is not immediate. The units of $\mathbf{R}\left(\left(\mathbf{G}^{\leq 0}\right)\right)$ are the elements of $\mathbf{R}$, so the irreducible elements (if any) are those series $a \in$ $\mathbf{R}\left(\left(\mathbf{G}^{\leq 0}\right)\right)$ which do not admit a factorization $a=b c$ with $b, c \notin \mathbf{R}$. The ring $\mathbf{R}\left(\left(\mathbf{G}^{\leq 0}\right)\right)$ is a standard part of $\mathbf{R}((\mathbf{G}))$ in the sense that every element of $\mathbf{R}((\mathbf{G}))$ is within infinitesimal distance from one and only one element of $\mathbf{R}((\mathbf{G} \leq 0))$. It is not true in general that a standard part of a real closed field necessarily has some irreducible element: Alex Wilkie noted that the Puiseux series with real coefficients form a real closed subfield of $\mathbf{R}((\mathbf{Q}))$ which has a standard part (and also an integer part) without irreducible elements. Such a standard part is given by the subring $\mathbf{R}\left[\mathbf{Q}^{\leq 0}\right]$ of $\mathbf{R}\left(\left(\mathbf{Q}^{\leq 0}\right)\right)$ consisting of the series with finite support. We thus have a nice example of a ring without irreducibles. Note that our $\operatorname{ring} \mathbf{R}\left(\left(\mathbf{G}^{\leq 0}\right)\right)$ contains a subring isomorphic to $\mathbf{R}\left[\mathbf{Q}^{\leq 0}\right]$ (by divisibility of $\mathbf{G}$ ).

The above considerations give us some (weak) evidence in favor of the absence of irreducibles. However [Conway 76] contains the following candidate for an irreducible element of $\mathbf{R}\left(\left(\mathbf{G}^{\leq 0}\right)\right.$ ) (Conway's group $\mathbf{G}$ is a proper class because he works inside the huge ring of "omnific integers", which is an integer part of the real closed field of "surreal numbers"):

$$
\sum_{n} t^{-1 / n}+1
$$

where $n$ ranges over the positive integers. (Conway did not have the minus sign in the exponents because of the change of variables $x^{1}=t^{-1}$, but then one has to consider anti-well-ordered supports.)

[Gonshor 86] obtains several partial results, some against the existence of irreducibles in $\mathbf{R}\left(\left(\mathbf{G}^{\leq 0}\right)\right)$, and some in favor. He points out the importance of the special case $\mathbf{G}=(\mathbf{R},+, 0, \leq)$ and shows how to reduce to it, in some cases, at the expense of expanding the field of coefficients $\mathbf{R}$. Gonshor's book contains the following quite different candidate for an irreducible series:

$$
t^{-\sqrt{2}}+t^{-1}+1
$$

which was also considered in Conway's lectures.

The choice of the exponents is motivated by the fact that if the support of an irreducible series is finite, then its exponents must be linearly independent over $\mathbf{Q}$, as otherwise a change of variables will transform the series into an ordinary polynomial of degree $>2$ over the reals, which is of course reducible. (This is 
the reason why the ring $\mathbf{R}\left[\mathbf{Q}^{\leq 0}\right]$ mentioned above has no irreducible elements.) [Biljacovic 96] showed that $t^{-\sqrt{2}}+t^{-1}+1$ is irreducible in the subring $K[\mathbf{G} \leq 0$ ] of $K\left(\left(\mathbf{G}^{\leq 0}\right)\right)$ consisting of all series with finite support. His results were preceded by a related result of [Moniri 94] which showed its irreducibility in a smaller ring.

Despite all these partial results the problem of the existence of irreducibles in $K((\mathbf{G} \leq 0))$ remained open, even in the special case $K=\mathbf{R}$ and $\mathbf{G}=(\mathbf{R},+, 0, \leq)$, or in the case of the ring of the omnific integers.

1.4. Related work on open induction. Quite independently of the work on omnific integers, similar problems about existence of irreducible elements arose in the context of the models of open induction. In particular, [Macintyre-Marker 89] asked: Is there a recursive model of open induction with infinite primes? The emphasis here was on the recursivness of the model (which implies countability), so the question is not the same as the one we consider in this paper (i.e. the existence of irreducible elements in the uncountable ring $K\left(\left(\mathbf{G}^{\leq 0}\right)\right)$ ). However the two problems are related: in both cases one is concerned with existence of irreducible elements in integer parts of real closed fields. A recursive model of open induction with infinite primes was constructed in [Berarducci - Otero 96] and independently by [Moniri 94] (and subsequently [Biljacovic 96]) in the already mentioned papers. Unlike the models constructed by Moniri and Biljacović, the one of Berarducci and Otero is based on an effective version of a theorem of [Wilkie 78] and has the further property of being "normal" (integrally closed in its fraction field). The resulting ring is a recursive integer part of the field of Puiseux series which is not truncation closed, so in this respect behaves differently from the rings considered in this paper.

1.5. Results. We prove that if $K$ is a field of characteristic zero (not even assumed to be orderable, e.g. the complex numbers) and $\mathbf{G}$ is an ordered abelian divisible group, then:

$K\left(\left(\mathbf{G}^{\leq 0}\right)\right)$ does have irreducible elements.

We first show this under the assumption that $\mathbf{G}$ is the ordered additive group $(\mathbf{R},+, 0, \leq)$ of real numbers. In an appendix we extend it to the general case using a suggestion of M.-H. Mourgues based on an idea of Gonshor, which allows us to reduce to the special case $\mathbf{G}=\mathbf{R}$ at the expense of enlarging the field of coefficients $K$. So it is important to work with a general $K$ and not only with $K=\mathbf{R}$.

We use the following criterion for irreducibility which depends only on the order type of the support.

(Theorem 10.5) Suppose that $a \in K\left(\left(\mathbf{R}^{\leq 0}\right)\right)$ is not divisible by any monomial $t^{\gamma}$ with $\gamma<0$. If the order type of the support of a is either $\omega$ or of the form $\omega^{\omega^{\beta}}$, then both a and $a+1$ are irreducible.

If $\mathbf{G}$ is archimedean, then it can be embedded in $\mathbf{R}$. So the above theorem holds for $K\left(\left(\mathbf{G}^{\leq 0}\right)\right)$ as well. In the non-archimedean case we can still prove the existence of irreducibles as follows:

(Theorem 12.1) Let $Q$ be an archimedean subgroup of $\mathbf{G}$ and suppose that $a \in K\left(\left(Q^{<0}\right)\right) \subset K\left(\left(\mathbf{G}^{<0}\right)\right)$ has support of order type $\omega$ or $\omega^{\omega^{\alpha}}(\alpha$ an ordinal) and $a$ is not divisible by any monomial $t^{\gamma}$ with $\gamma \in Q^{<0}$. Then $a+1$ is irreducible in $K\left(\left(\mathbf{G}^{\leq 0}\right)\right)$.

In particular Conways's series $\sum_{n} t^{-1 / n}+1$ is irreducible (in the non-archimedean case we do need to add 1 , as otherwise the series is divisible by a monomial). We 
can allow $\mathbf{G}$ to be a proper class, but restricting to series whose support is a set. So Conway's series is irreducible even in the ring of omnific integers.

The ordinals which appear in our criterion are exactly the infinite ordinals which cannot be obtained as a sum or product of two smaller ordinals: so what our criterion says is that if the order type is irreducible the series is irreducible.

The criterion does not apply to the series $t^{-\sqrt{2}}+t^{-1}+1$. To handle it we instead show:

(Theorem 11.2) Any irreducible element of $K\left[\mathbf{R}^{\leq 0}\right]$ remains irreducible in $K((\mathbf{R} \leq 0))$.

We have not checked whether the above theorem holds for a general $\mathbf{G}$ instead of $\mathbf{R}$. Combined with Biljacović's work, this shows that $t^{-\sqrt{2}}+t^{-1}+1$ is irreducible in $K((\mathbf{R} \leq 0))$.

Both results are based on a new kind of valuation which we now describe.

1.6. A new kind of valuation taking ordinal numbers as values. Let $O R$ be the class of all ordinal numbers. Consider the order type map

$$
\text { ot }: K((\mathbf{G})) \rightarrow O R
$$

which assigns to each series the order type of its support. If $\mathbf{G}=\mathbf{R}$, then ot has image contained in the countable ordinals. This map does not have good algebraic properties; for instance, there is no way of predicting the order type of a product $b c$ given the order types of $b$ and $c$ (e.g. take $c=b^{-1}$ ). However we will be able to show that if we restrict this map to the subring $K((\mathbf{G} \leq 0)) \subset K((\mathbf{G}))$ (and we assume $\mathbf{G}=\mathbf{R}$ ), then the order type of the product is "roughly" the product of the order types, where however we need to consider not the usual product of ordinals but a commutative variant of it known as "natural product" or "Hessemberg's product" [Hausdorff 27], which we write as $\odot$. The precise result is:

(Corollary 9.9) Let $b, c \in K\left(\left(\mathbf{R}^{\leq 0}\right)\right)$. Then ot $(b c)=$ ot $(b) \odot$ ot $(c)$ provided there are ordinals $\beta$ and $\xi$ with ot $(b)=\omega^{\beta}$ and ot $(c)=\omega^{\xi}$.

The natural product satisfies the property $\omega^{n} \odot \omega^{m}=\omega^{n+m}$ whenever $n, m$ are natural numbers. In general $\omega^{\alpha} \odot \omega^{\beta}=\omega^{\alpha \oplus \beta}$, where $\oplus$ is the "natural sum" of ordinals.

The above theorem puts restrictions on the order types of the possible factors of an element of $K\left(\left(\mathbf{G}^{\leq 0}\right)\right)$, and it will be the key ingredient for the proof of the existence of irreducible elements (some extra work is however needed). The fact that the theorem holds only for order types of the form $\omega^{\alpha}$ is not very restrictive, due to the fact that every ordinal has a final segment of type $\omega^{\alpha}$ (and the final part of a product of two series depends only on the final parts of the factors). Some remarks are in order:

1. It is not difficult to prove $o t(b c) \leq o t(b) \odot o t(c)$. This holds without any restriction on the order types of $b, c$ (and we can even allow $b, c$ to range over the whole field $K((\mathbf{G}))$ ).

2. It is not too difficult to prove the theorem for series with positive coefficients, because in that case there cannot be "cancellations" in the product.

3. To prove $o t(b c)=o t(b) \odot o t(c)$ (with the stated restrictions) we need to prove that there are "few cancellations" in the product of two elements of $K((\mathbf{G} \leq 0))$ (this is false in the field $K((\mathbf{G}))$ : take $c=b^{-1}$ ). 
It is convenient to derive Corollary 9.9 from a more elegant, although essentially equivalent statement asserting the existence of a suitable valuation. We need some preliminaries.

As usual let $\mathbf{G}$ be an ordered divisible abelian group. Let $J \subset K\left(\left(\mathbf{G}^{\leq 0}\right)\right)$ be the ideal generated by the set of monomials $\left\{t^{\gamma} \mid \gamma \in \mathbf{G}^{<0}\right\}$. An element is in $J$ iff it is divisible by a monomial, so all the elements of $J$ are trivially reducible. Note that $J$ is not maximal, since it is properly contained in the maximal ideal $\mathcal{M} \subseteq K\left(\left(\mathbf{G}^{\leq 0}\right)\right)$ consisting of all series without constant term. We clearly have

$$
K\left(\left(\mathbf{G}^{\leq 0}\right)\right) / J \supseteq K\left(\left(\mathbf{G}^{\leq 0}\right)\right) / \mathcal{M}=K
$$

The elements of $K\left(\left(\mathbf{G}^{\leq 0}\right)\right) / J$ can be thought of as germs of power series. Two series (both not in $J$ ) have the same germ if they have a common final part.

We will define a map called ordinal-value

$$
v_{J}: K\left(\left(\mathbf{G}^{\leq 0}\right) \rightarrow O R\right.
$$

which is similar to the order type map ot but only assumes values of the form $\omega^{\alpha}$ or 0 . If $b$ has limit order type and does not belong to $J$, then $v_{J}(b)$ is the order type of a sufficiently small final segment of $b$. For the general case see Definition 5.2. The definition of $v_{J}$ makes sense for an arbitrary $\mathbf{G}$, but to prove that it has good algebraic properties we specialize to the case $\mathbf{G}=\mathbf{R}$. In this case we only need countable ordinals, and we can prove (Lemma 5.5 and Theorem 9.7):

1. $v_{J}(b)=0$ iff $b \in J$

2. $v_{J}(b+c) \leq \max \left\{v_{J}(b), v_{J}(c)\right\}$,

3. $v_{J}(b c)=v_{J}(b) \odot v_{J}(c)$ (multiplicative property).

The proof of the multiplicative property is the most difficult result of this paper, and the irreducibility results can be derived from it. It implies in particular that the ideal $J$ is prime. The multiplicative property is easily seen to be equivalent to Corollary 9.9, which only speaks about ordinal types and does not mention $v_{J}$. Since $b \equiv c \bmod J$ implies $v_{J}(b)=v_{J}(c)$, we can pass to the quotient and obtain a map

$$
v_{J}: K\left(\left(\mathbf{G}^{\leq 0}\right) / J \rightarrow O R\right.
$$

which satisfies 2, 3 and $v_{J}(x)=0$ iff $x=0$ (where $x \in K\left(\left(\mathbf{G}^{\leq 0}\right) / J\right)$. Thus $v_{J}$ is an non-archimedean absolute value on $K\left(\left(\mathbf{R}^{\leq 0}\right)\right) / J$ except for the fact that usually absolute values are defined on fields, and $K((\mathbf{R} \leq 0)) / J$ is only a domain. There is however no problem in extending $v_{J}$ to the fraction field of $K\left(\left(\mathbf{R}^{\leq 0}\right)\right) / J$ (but we will not need this): to do that we first observe that $(O R, \oplus, \odot, \leq)$ is contained in an ordered field, for instance the one defined in [Sikorski 48], or the larger real closed field of surreal numbers [Conway 76, Note on page 28]. So if we define $v_{J}(b / c)=v_{J}(b) / v_{J}(c)$ we obtain an absolute value into the surreal numbers.

Remark 1.1. Using the fact that $v_{J}$ has image contained in $\left\{\omega^{\alpha} \mid \alpha\right\} \cup\{0\}$, we can define a new map $w_{J}: K((\mathbf{R} \leq 0)) / J \rightarrow O R \cup\{-\infty\}$ which behaves like a polynomial degree: $\omega^{w_{J}(b)}=v_{J}(b)$ (with $w_{J}(b)=-\infty$ if $\left.v_{J}(b)=0\right)$. We have $w_{J}(b c)=$ $w_{J}(b) \oplus w_{J}(c)$ and $w_{J}(b+c) \leq \max \left\{w_{J}(b), w_{J}(c)\right\}$. We can obtain a Krull valuation [Endler 72] by reversing the order.

Remark 1.2. $K\left(\left(\mathbf{R}^{\leq 0}\right)\right) / J$ is not a valuation ring: there is a non-zero element $x$ of its fraction field such that neither $x$ nor $x^{-1}$ belongs to $K\left(\left(\mathbf{R}^{\leq 0}\right)\right) / J$, for instance 
$x=(b+J) /(c+J)$ where $b:=\sum_{n} t^{-1 / n}$ and $c:=\sum_{n} t^{-1 / n^{2}}$. This can be easily established with the help of the valuation $v_{J}$.

\section{OUtline of the Proof of the MUltiplicative Property \\ OF THE ORDINAL-VALUE}

In this section we give a brief qualitative sketch of the proof of the multiplicative property.

It is easy to show that $v_{J}$ is submultiplicative: $v_{J}(b c) \leq v_{J}(b) \odot v_{J}(c)$. This corresponds to the fact that the order type of a product $b c$ is less than or equal to the natural product of the order types of $b$ and $c$.

The idea is to prove the multiplicative property $v_{J}(b c)=v_{J}(b) \odot v_{J}(c)$ by induction on the ordinals making use of the submultiplicative property. It is actually more convenient to prove the multiplicative property for several factors:

$$
v_{J}\left(b_{0}^{k_{0}} \cdot \ldots \cdot b_{n}^{k_{n}}\right)=\bigodot^{k_{0}} v_{J}\left(b_{0}\right) \odot \ldots \odot \bigodot^{k_{n}} v_{J}\left(b_{n}\right)
$$

where $b_{i} \in K\left(\left(\mathbf{R}^{\leq 0}\right)\right)$ and $k_{i} \in \mathbf{N}$. This is proved by induction on a suitable notion of complexity of the formal expressions $b_{0}^{k_{0}} \cdot \ldots \cdot b_{n}^{k_{n}}$. In the sequel we write $\equiv$ for congruence modulo the ideal $J$.

I. Given $\gamma \in \mathbf{G}$ (later we specialize to $\mathbf{G}=\mathbf{R}$ ) and $b \in K((\mathbf{G} \leq 0)$ ), we will define (Definition 6.1) a new series $b^{\mid \gamma} \in K\left(\left(\mathbf{G}^{\leq 0}\right)\right.$ ) in such a way that $b^{\mid \gamma} \equiv c^{\mid \gamma} \bmod J$ iff $b, c$ coincide near $\gamma$ (in the sense that if $\delta$ is sufficiently close to $\gamma$ then the coefficients of $t^{\delta}$ in $b, c$ coincide). The series $b^{\mid \gamma}$ is obtained by truncating $b$ at $\gamma$ and multiplying the resulting series by the normalization factor $t^{-\gamma}$. We call $b^{\mid \gamma}+J$ the germ at $\gamma$ of $b$. Assuming $\mathbf{G}=\mathbf{R}$, we will prove (Lemma 7.5) the following convolution formula which shows that these germs at $\gamma$ behave like generalized coefficients:

$$
(b c)^{\mid \gamma} \equiv \sum_{\beta+\xi=\gamma} b^{\mid \beta} c^{\mid \xi} \bmod J
$$

Equation (1) holds for more general groups $\mathbf{G}$, but we will not need this fact.

II. The multiplicative property says that $v_{J}(b c)$ is as big as possible. The plan is to show that $v_{J}(b c)$ is "big" by showing that $v_{J}\left((b c)^{\mid \gamma}\right)$ is "big" for "many" values of $\gamma$. We try to use the convolution formula to compute $(b c)^{\mid \gamma}$.

III. If $\gamma$ is chosen in a suitable way with respect to the support of $b$, we can isolate the most significant terms in the right-hand-side of equation (1) and obtain (Lemma 7.7):

$$
\begin{aligned}
(b c)^{\mid \gamma} \equiv & b^{\mid \gamma} c+b c^{\mid \gamma} \\
& + \text { terms of small value }
\end{aligned}
$$

where "small" means smaller than the "expected" ordinal-value of $b^{\mid \gamma} c$, namely $v_{J}\left(b^{\mid \gamma}\right) \odot v_{J}(c)$. Of course until we have proved the multiplicative property of $v_{J}$ we do not know whether the expected ordinal-value coincides with the actual ordinalvalue. Equation (2) says that in some sense $b \mapsto b^{\mid \gamma}$ behaves like a derivation. We do not enter into the details of how $\gamma$ is to be chosen; let us just remark that it is chosen inside a set $X(b)$ depending on $b$, so we introduce an asymmetry between $b$ and $c$.

IV. Special cases. If we knew that $v_{J}\left(b c^{\mid \gamma}\right)<v_{J}\left(b^{\mid \gamma} c\right)$ we could deduce $v_{J}\left((b c)^{\mid \gamma}\right)$ $=v_{J}\left(b^{\mid \gamma} c\right)$ and try an inductive argument in the form: if for many $\gamma^{\prime} s b^{\mid \gamma} c$ has its 
expected ordinal-value, then $b c$ has its expected ordinal-value. Another favorable case is when $b=c$. In this case we obtain:

$$
\begin{aligned}
\left(b^{2}\right)^{\mid \gamma} \equiv & 2 b^{l \gamma} b \\
& + \text { terms of small value }
\end{aligned}
$$

V. In the general case we can assume that $b$ has "principal value" (Definition 6.4) smaller than or equal to that of $c$. (This is a technical assumption that can always be ensured by interchanging the roles of $b, c$.) The crucial idea of the whole proof is to multiply both sides of equation (2) by $c$, obtaining:

$$
\begin{aligned}
c(b c)^{\mid \gamma} \equiv & b^{\mid \gamma} c^{2}+b c c^{\mid \gamma} \\
& + \text { terms of small value }
\end{aligned}
$$

where now "small" means "smaller than the expected value of $b^{\mid \gamma} c^{2}$ ", namely $\leq$ $v_{J}\left(b^{\mid \gamma}\right) \odot v_{J}(c) \odot v_{J}(c)$. Apparently we have only introduced a complication, but the advantage is that now the expression $b c$ appears on both sides of $\equiv$. For the sake of proving that $b c$ has big ordinal-value we can as well suppose the contrary. Then the second term on the right-hand-side becomes small and we obtain:

$$
\begin{aligned}
c(b c)^{\mid \gamma} \equiv & b^{\mid \gamma} c^{2} \\
& + \text { terms of small value }
\end{aligned}
$$

A similar argument yields for every positive integer $k$ the equation:

$$
\begin{aligned}
c\left(b^{k} c\right)^{\mid \gamma} \equiv & b^{\mid \gamma} b^{k-1} c^{2} \\
& + \text { terms of small value }
\end{aligned}
$$

VI. To carry out an inductive proof based on equation (3) we define a notion of complexity such that the expression $b^{\mid \gamma} b^{k-1} c^{2}$ is "simpler" than $b^{k} c$. This seems almost paradoxical already in the case $k=1$, but remember that $b$ and $c$ do not play a symmetrical role in our arguments since $b$ was assumed to have "smaller or equal principal value". We have to arrange things so that in passing from $b^{k} c$ to $b^{\mid \gamma} b^{k-1} c^{2}$, the complication introduced by the substitution $c \mapsto c^{2}$ is more than compensated by the "simplification" $b^{k} \mapsto b^{\mid \gamma} b^{k-1}$ (it is reasonable to consider the term $b^{\mid \gamma}$ simpler than $b$, because it has smaller ordinal value provided $\gamma$ is sufficiently small). This suggests that $b$ should count more than $c$. The actual definition of complexity (Definition 9.3) is however rather complicated, due to the apparently conflicting requirements that $b$ has principal value smaller than or equal to that of $c$.

VII. Once the correct definition of complexity has been given, we show that

$$
v_{J}\left(b_{0}^{k_{0}} \cdot \ldots \cdot b_{n}^{k_{n}}\right)=\bigodot^{k_{0}} v_{J}\left(b_{0}\right) \odot \ldots \odot \bigodot^{k_{n}} v_{J}\left(b_{n}\right)
$$

by induction on the complexity. To this aim we write $b_{0}^{k_{0}} \cdot \ldots \cdot b_{n}^{k_{n}}=b^{k} c$ by suitably selecting one particular $b_{i}$ to play the role of $b$ in equation (3). Assuming inductively that the displayed term in the right-hand-side of equation (3) has its expected ordinal-value, we infer from the equation the ordinal-value of $c\left(b^{k} c\right)^{\mid \gamma}$ and by varying $\gamma$ we deduce the ordinal-value of $c\left(b^{k} c\right)$. Using the submultiplicative property of $v_{J}$, we can now divide by the extra factor $c$ which has been introduced during the proof and obtain the ordinal-value of the original expression $b^{k} c$, thus completing the induction.

We now come to the precise definitions and the technical details. 


\section{NATURAL SUM AND PRODUCT OF ORDINALS}

We denote by $O R$ the class of all ordinals and by LIM the class of all limit ordinals. The ordinal sum $\alpha+\beta$, ordinal product $\alpha \cdot \beta$ and ordinal exponentiation $\alpha^{\beta}$ are defined by induction on their second argument and are continuous in their second argument: $\alpha+0=\alpha, \alpha+(\beta+1)=(\alpha+\beta)+1$ and $\alpha+\lambda=\sup _{\xi<\lambda} \alpha+\xi$ for $\lambda \in L I M$; similarly for $\alpha \cdot \beta$ and $\alpha^{\beta}$. The product $\alpha \cdot \beta$ will also be written as $\alpha \beta$.

Definition 3.1. An ordinal $\rho$ is additive principal if it cannot be written as the ordinal sum of two ordinals strictly smaller than $\rho$. Let $\mathbf{H}$ be the class of all additive principal ordinals.

Fact 3.2. 1. $\rho \in \mathbf{H}$ iff for every $\alpha, \beta<\rho, \alpha+\beta<\rho$,

2. $\rho \in \mathbf{H}$ iff either $\rho=0$ or there is $\alpha$ such that $\rho=\omega^{\alpha}$

If an ordinal is not additive principal it can be written as a sum of two smaller ordinals. Since this process must end, every ordinal is a finite sum of additive principal ordinals. Thus we have:

Fact 3.3. (Cantor normal form) For every ordinal $\alpha \neq 0$, there are uniquely determined ordinals $\alpha_{1} \geq \ldots \geq \alpha_{n}$ such that $\alpha=\omega^{\alpha_{1}}+\ldots+\omega^{\alpha_{n}}$. The right-hand side is the Cantor normal form of $\alpha$, and we call $\omega^{\alpha_{n}}$ the principal part of $\alpha$.

The exponents $\alpha_{i}$ appearing in the Cantor normal form of $\alpha$ are not necessarily strictly smaller than $\alpha$, for instance there are many ordinals satisfying $\alpha=\omega^{\alpha}$. $\left(\omega_{1}\right.$ has this property, and also many countable ordinals.) A fact that we will repeatedly use is that every sufficiently small final segment of a non-zero ordinal $\alpha$ has order type equal to the principal part of $\alpha$.

Definition 3.4. The natural sum $\oplus$ and natural product $\odot$ of two ordinals are commutative variants of the ordinal sum + and ordinal product · (see [Hausdorff 27, p. 68] or [Pohlers 80]). To define the natural sum of two non-zero ordinals we consider the Cantor normal forms $\alpha=\omega^{\alpha_{1}}+\ldots+\omega^{\alpha_{n}}$ and $\beta=\omega^{\alpha_{n+1}}+\ldots+\omega^{\alpha_{n+m}}$, and we set $\alpha \oplus \beta=\omega^{\alpha_{\pi(1)}}+\ldots+\omega^{\alpha_{\pi(n+m)}}$, where $\pi$ is a permutation of the integers $1, \ldots, n+m$ such that $\alpha_{\pi(1)} \geq \ldots \geq \alpha_{\pi(n+m)}$. If $\alpha=0$ we set $\alpha \oplus \beta=\beta \oplus \alpha=\beta$. The natural product is first defined on $\mathbf{H}$ by: $\omega^{\alpha} \odot \omega^{\beta}=\omega^{\alpha \oplus \beta}$ and $\gamma \odot 0=$ $0 \odot \gamma=0$. We then extend $\odot$ to all the ordinals using the Cantor normal form and distributivity: $\gamma \odot(\alpha \oplus \beta)=(\alpha \oplus \beta) \odot \gamma=(\alpha \odot \gamma) \oplus(\beta \odot \gamma)$.

Clearly $\alpha \cdot \beta \leq \alpha \odot \beta$ and $\alpha+\beta \leq \alpha \oplus \beta$. We now prove that $\oplus$ and $\odot$ are strictly increasing:

Lemma 3.5. 1. If $\alpha<\beta$, then $\alpha \oplus \gamma<\beta \oplus \gamma$.

2. If $\alpha<\beta$, then $\alpha \odot \gamma<\beta \odot \gamma$ provided $\gamma \neq 0$.

Proof. 1. Consider two Cantor normal forms $\alpha=\omega^{\alpha_{1}}+\ldots+\omega^{\alpha_{n}}$ and $\beta=\omega^{\beta_{1}}+$ $\ldots+\omega^{\beta_{m}}$. Then $\alpha<\beta$ iff and only if either $\alpha_{1}<\beta_{1}$ or $\alpha_{1}=\beta_{1}$ and $\omega^{\alpha_{2}}+$ $\ldots+\omega^{\alpha_{n}}<\omega^{\beta_{2}}+\ldots+\omega^{\beta_{m}}$. In other words, to compare two ordinals we compare lexicographically the exponents in their Cantor normal forms. Point 1 easily follows.

2. Since $\odot$ distributes over $\oplus$ and the latter is strictly increasing, in order to show that $\odot$ is strictly increasing we can reduce to the following case: if $\omega^{\alpha}<\omega^{\beta}$, then $\omega^{\alpha} \odot \omega^{\gamma}<\omega^{\beta} \odot \omega^{\gamma}$. By definition of $\odot$ this means $\omega^{\alpha \oplus \gamma}<\omega^{\beta \oplus \gamma}$. Now use the fact that both $\oplus$ and $x \mapsto \omega^{x}$ are strictly increasing. 
Definition 3.6. MP is the class of all the multiplicative principal ordinals, namely the class of all $\alpha \in O R$ such that $\alpha>0$ and for every $\beta, \gamma<\alpha$ we have $\beta \gamma<\alpha$.

For the following results see [Pohlers 80].

Fact 3.7. Suppose $\beta, \gamma<\alpha$. If $\alpha \in \mathbf{H}$, then $\beta \oplus \gamma<\alpha$. If $\alpha \in \mathbf{M P}$, then $\beta \odot \gamma<\alpha$.

Fact 3.8. $\rho \in \mathbf{M P}$ if and only if $\rho=1$ or $\rho$ is of the form $\omega^{\omega^{\alpha}}$.

\section{WELL-ORDERED SUBSETS OF AN ORDERED GROUP}

We are interested in the order types of the well-ordered subsets of an ordered group, and their behavior under unions and the group operation. Let $\mathbf{G}=(\mathbf{G},+$, $0,<)$ be an ordered abelian group. In the special case $\mathbf{G}=\mathbf{R}$ the order types of the well-ordered subsets of $\mathbf{G}$ are exactly the countable ordinals. In the sequel $B, C, \ldots$ denote well-ordered subsets of $\mathbf{G}$. We write $B \leq \gamma$ if all elements of $B$ are $\leq \gamma$. Thus $\sup B=\gamma$ iff $B \leq \gamma$ and $\forall \beta<\gamma, B \not \leq \beta$. Since we do not (yet) assume that $\mathbf{G}$ is complete, the supremum might not exist.

Lemma 4.1. $\operatorname{ot}(B \cup C) \leq \operatorname{ot}(B) \oplus \operatorname{ot}(C)$.

Proof. We can assume $B, C$ disjoint; otherwise we can make them disjoint by replacing $B$ with a smaller set without changing the union $B \cup C$. We proceed by induction on $o t(B \cup C)$. If $o t(B \cup C)=0$ there is nothing to prove. If $o t(B \cup C)=\alpha+1$, let $a \in B \cup C$ be the last element of $B \cup C$. Without loss of generality suppose $a \in C$ and let $C^{\prime}=C \backslash\{a\}$. Then $a \notin B$ and therefore ot $(B \cup C)=o t\left(B \cup C^{\prime}\right)+1$. By induction ot $\left(B \cup C^{\prime}\right) \leq$ ot $(B) \oplus$ ot $\left(C^{\prime}\right)$. So ot $(B \cup C) \leq$ ot $(B) \oplus$ ot $\left(C^{\prime}\right)+1=\operatorname{ot}(B) \oplus$ ot $(C)$.

Limit case. Suppose ot $(B \cup C)=\lambda \in L I M$. For $\beta<\lambda$ let $H_{\beta}$ be the initial segment of $B \cup C$ of order type $\beta$, and let $B_{\beta}=B \cap H_{\beta}$ and $C_{\beta}=C \cap H_{\beta}$. By induction $\beta=\operatorname{ot}\left(B_{\beta} \cup C_{\beta}\right) \leq \operatorname{ot}\left(B_{\beta}\right) \oplus \operatorname{ot}\left(C_{\beta}\right) \leq \operatorname{ot}(B) \oplus$ ot $(C)$. Hence $\lambda=$ $\sup _{\beta<\lambda} \beta \leq$ ot $(B) \oplus$ ot $(C)$.

Lemma 4.2. If $B$ has limit order type and $B \leq 0$, then for every proper initial segment $H$ of $B$ there is $\gamma<0$ such that $H \leq \gamma$.

Proof. Let $\gamma \in B, \gamma \notin H$. Since $o t(B) \in L I M, \gamma<0$.

Lemma 4.3. If $\sup B=\sup C=0$ and $B, C$ have additive principal order type $>1$, then ot $(B \cup C)=\max \{\operatorname{ot}(B)$, ot $(C)\}$.

Proof. Let $\rho=\max \{o t(B), o t(C)\}$. It suffices to show that every proper initial segment of $B \cup C$ has order type $<\rho$. By the previous result, any such segment is contained in the union of a proper initial segment $B^{\prime}$ of $B$ and a proper initial segment $C^{\prime}$ of $C$. Now ot $\left(B^{\prime} \cup C^{\prime}\right) \leq \operatorname{ot}\left(B^{\prime}\right) \oplus$ ot $\left(C^{\prime}\right)<\rho($ as $\rho \in \mathbf{H})$.

Definition 4.4. $B+C=\{x+y \mid x \in B, y \in C\}$.

Lemma 4.5. ot $(B+C) \leq o t(B) \odot$ ot $(C)$.

Proof. By induction on ot $(B) \odot$ ot $(C)$.

Special case. Suppose that ot $(B)$ and $o t(C)$ are additive principal. It is enough to show that if $H$ is a proper initial segment of $B+C$, then $H$ has order type $<$ ot $(B) \odot$ ot $(C)$. Let $\beta \in B$ and $\gamma \in C$ be such that $\beta+\gamma \notin H$. Let $B^{\prime}$ be the set of elements of $B$ which are $<\beta$, and let $C^{\prime}$ be the set of elements of $C$ which are $<\gamma$. Then $H \subseteq\left(B^{\prime}+C\right) \cup\left(B+C^{\prime}\right)$. By induction ot $\left(B^{\prime}+C\right) \leq$ 
ot $\left(B^{\prime}\right) \odot$ ot $(C)$ and $o t\left(B+C^{\prime}\right) \leq o t(B) \odot$ ot $\left(C^{\prime}\right)$. Since the order type of the union of two sets is bounded by the natural sum of the respective order types, $o t(H) \leq\left(o t\left(B^{\prime}\right) \odot o t(C)\right) \oplus\left(o t(B) \odot o t\left(C^{\prime}\right)\right)$. The two terms of this natural sum are $<o t(B) \odot o t(C)$, so their natural sum is also $<o t(B) \odot(C)$ since this latter ordinal is additive principal (as the additive principal ordinals are closed under natural product).

General case. Let $\operatorname{ot}(B)=\omega^{\beta_{1}}+\cdots+\omega^{\beta_{n}}$ be the Cantor normal form of ot $(B)$ and let $o t(C)=\omega^{\gamma_{1}}+\cdots+\omega^{\gamma_{k}}$ be the Cantor normal form of $C$. We can write $B=\bigcup_{i=1}^{n} B_{i}$ with $o t\left(B_{i}\right)=\omega^{\beta_{i}}$ and $C=\bigcup_{j=1}^{k} C_{j}$ with ot $\left(C_{j}\right)=\omega^{\gamma_{j}}$. Then $B+C=$ $\bigcup_{i, j} B_{i}+C_{j}$ and by the special case and induction ot $\left(B_{i}+C_{j}\right) \leq \operatorname{ot}\left(B_{i}\right) \odot$ ot $\left(C_{j}\right)$. The order type of the union of finitely many sets is bounded by the natural sum of the respective order types. So $o t(B+C)$ is bounded by the natural sum of the various $o t\left(B_{i}\right) \odot o t\left(C_{j}\right)$, and this natural sum coincides with $o t(B) \odot$ ot $(C)$.

For later purposes we need:

Lemma 4.6. If $\lambda$ is a limit ordinal, then $\lambda$ coincides with the order type of the set $I \subseteq \lambda$ of all successor ordinals $\beta<\lambda$.

Proof. Since $\lambda \in L I M$, we can consider the successor function $s: \lambda \rightarrow \lambda, x \mapsto$ $x+1$. Since $s$ is strictly increasing, the image of $s$ has the same order type as its domain.

Lemma 4.7. Let $\lambda$ be a limit ordinal and let $\left\{B_{i} \mid i<\lambda\right\}$ be a family of wellordered subsets of $\mathbf{G}$ with the property that if $i<j<\lambda$, then $B_{j}$ has an element bigger than all elements of $B_{i}$. Suppose that for each $i<\lambda$ every non-empty final segment of $B_{i}$ has order type $\geq \rho$. If $\bigcup_{i<\lambda} B_{i}$ is well ordered, then it has order type $\geq \rho \cdot \lambda$.

Proof. Let $I$ be the set of all successor ordinals less than $\lambda$. Then $\operatorname{ot}(I)=\lambda$ by Lemma 4.6. The ordinal $\rho \cdot$ ot $(I)$ can be realized as the order type with respect to the lexicographic ordering of the set $I \times \rho$ consisting of all pairs $(i, \beta)$ with $i \in I$ and $\beta<\rho$ (to compare two such pairs we compare the first components, and if they are equal we compare the second ones). For $i \in I$ let $T_{i}$ be the subset of $B_{i}$ consisting of all the elements $x \in B_{i}$ with $x \geq B_{j}$ for all $j<i$. Since each $i \in I$ has an immediate predecessor $j<\lambda, T_{i}$ is a non-empty final segment of $B_{i}$ and therefore it has order type $\geq \rho$. To finish the proof, consider the strictly increasing map from $I \times \rho$ into $\bigcup_{i<\lambda} B_{i}$ sending $(i, \beta)$ into the $\beta$-th element of $T_{i}$.

\section{The ORDinAL-VALUe of A GENERALIZED POWER SERIES}

Definition 5.1. Let $J \subset K\left(\left(\mathbf{G}^{\leq 0}\right)\right)$ be the ideal generated by all the monomials $t^{\gamma}$ with $\gamma<0$. We call the elements of the quotient ring $K((\mathbf{G} \leq 0)) / J$ germs of power series.

Clearly $J$ consists of all the series with negative support bounded away from zero: $b \in J$ iff $\exists \gamma \in \mathbf{G}$ such that $S_{b} \leq \gamma<0$. Two series $b, c \in K\left(\left(\mathbf{G}^{\leq 0}\right)\right)$ have the same germ iff for every $\gamma \leq 0$ sufficiently close to zero, the coefficients of $t^{\gamma}$ in $b$ and $c$ coincide. In particular, for $\gamma$ sufficiently close to zero, $\gamma \in S_{b}$ iff $\gamma \in S_{c}$.

Definition 5.2. Given $b \in K\left(\left(\mathbf{G}^{\leq 0}\right)\right)$, we define its order type $o t(b) \in O R$ as the order type of the support $S_{b}$ of $b$. Let $J+K$ be the additive subgroup of $K\left(\left(\mathbf{G}^{\leq 0}\right)\right)$ generated by the ideal $J$ and the additive subgroup $K$. We write as 
usual $c \equiv b \bmod J+K$ for $b-c \in J+K$. We define the ordinal-value (or value) $v_{J}: K\left(\left(\mathbf{G}^{\leq 0}\right)\right) \rightarrow O R$ as follows.

1. $v_{J}(b)=0$ iff $b \in J$.

2. $v_{J}(b)=1$ iff $b$ is congruent to a non-zero element of $K$ modulo $J$.

3. $v_{J}(b)=\min \{o t(c) \mid c \equiv b \bmod J+K\}$ in the remaining cases.

A more geometrical definition of $v_{J}$ can be obtained as follows. For $\varepsilon \in \mathbf{G}^{>0}$ let $(-\varepsilon, 0)$ be the interval $\{x \in \mathbf{G} \mid-\varepsilon<x<0\}$ and let $B_{\varepsilon}$ be the intersection of the support $S_{b}$ of $b$ with $(-\varepsilon, 0)$. If there is some $\varepsilon$ with $B_{\varepsilon}=\emptyset$, then $v_{J}(b)$ is 1 or 0 depending on whether 0 is in the support of $b$ or not. If instead for every $\varepsilon$ the set $B_{\varepsilon}$ is non-empty, then there must be some $\varepsilon$ such that for every smaller $\varepsilon^{\prime}$ the sets $B_{\varepsilon}$ and $B_{\varepsilon^{\prime}}$ have the same order type. We then say that $B_{\varepsilon}$ is a stable interval for $b$. The order type of any stable interval is the ordinal-value of $b$, and it coincides with the principal part of the Cantor normal form of ot $(b)$ provided $o t(b)$ is a limit ordinal and the supremum of $S_{b}$ is 0 . Note that we consider the open interval $(-\varepsilon, 0)$ rather than the half-open one $(-\varepsilon, 0]$ which would seem more natural. This ensures the validity of the following remark and corresponds to the fact that in clause 3 we work modulo $J+K$ rather than modulo $J$.

Remark 5.3. The map $v_{J}: K\left(\left(\mathbf{G}^{\leq 0}\right)\right) \rightarrow O R$ has image contained in $\mathbf{H}$.

Remark 5.4. Given $b, c \in K((\mathbf{G}))$, the support of $b+c$ is included in the union $S_{b} \cup S_{c}$ of the respective supports, and the support of $b c$ is contained in $S_{b}+S_{c}$. By Lemma 4.1 and Lemma 4.4 we obtain:

1. $o t(b+c) \leq o t(b) \oplus o t(c)$,

2. $o t(b c) \leq o t(b) \odot o t(c)$.

We are now ready to prove the submultiplicative property of $v_{J}$. Part 2 of the following lemma will later be superseded by the proof of the multiplicative property.

Lemma 5.5. 1. $v_{J}(b+c) \leq \max \left\{v_{J}(b), v_{J}(c)\right\}$, with equality holding if $v_{J}(b) \neq$ $v_{J}(c)$.

2. $v_{J}(b c) \leq v_{J}(b) \odot v_{J}(c)$ (submultiplicative property).

Proof. 1. We assume $v_{J}(b)>1, v_{J}(c)>1$. In the remaining cases the proof is trivial. There are $b^{\prime} \equiv b \bmod J+K$ and $c^{\prime} \equiv c \bmod J+K$ such that $v_{J}(b)=o t\left(b^{\prime}\right)=$ $v_{J}\left(b^{\prime}\right)$ and $v_{J}(c)=\operatorname{ot}\left(c^{\prime}\right)=v_{J}\left(c^{\prime}\right)$. The support of $b^{\prime}+c^{\prime}$ is contained in the union of the supports of $b^{\prime}$ and $c^{\prime}$. Hence by Lemma 4.3 ot $\left(b^{\prime}+c^{\prime}\right) \leq \max \left\{o t\left(b^{\prime}\right), o t\left(c^{\prime}\right)\right\}$. Since $b+c \equiv b^{\prime}+c^{\prime} \bmod J+K$, if $v_{J}(b+c)>1$ then $v_{J}(b+c) \leq o t\left(b^{\prime}+c^{\prime}\right)$. So in any case $v_{J}(b+c) \leq \max \left\{v_{J}(b), v_{J}(c)\right\}$.

To finish the proof of 1 , assume further that $v_{J}(c)<v_{J}(b)$. Let $H$ be the set of elements in the support of $b^{\prime}$ which do not lie in the support of $c^{\prime}$. The support of $b^{\prime}+c^{\prime}$ contains $H$, so ot $\left(b^{\prime}+c^{\prime}\right) \geq \operatorname{ot}(H)$.

We claim that $o t(H)=o t\left(b^{\prime}\right)$. If this is not so, then the support of $b^{\prime}$ is the union of two sets of order type strictly smaller than ot $\left(b^{\prime}\right)(H$ and the support of $\left.c^{\prime}\right)$. This contradicts $o t\left(b^{\prime}\right)=v_{J}(b) \in \mathbf{H}$, and the claim is proved.

It follows that $o t\left(b^{\prime}+c^{\prime}\right) \geq \operatorname{ot}\left(b^{\prime}\right)$, and by Lemma 4.3 ot $\left(b^{\prime}+c^{\prime}\right)=o t\left(b^{\prime}\right) \in \mathbf{H}$. Hence ot $\left(b^{\prime}+c^{\prime}\right)=v_{J}\left(b^{\prime}+c^{\prime}\right)$. The congruence $b+c \equiv b^{\prime}+c^{\prime} \bmod J+K$ implies $v_{J}(b+c)>1$, and therefore $v_{J}(b+c)=v_{J}\left(b^{\prime}+c^{\prime}\right)=o t\left(b^{\prime}+c^{\prime}\right)=\operatorname{ot}\left(b^{\prime}\right)=v_{J}(b)$, as desired. 
2. We use the fact that the support of $b c$ is included in $S_{b}+S_{c}$. In the special case when $v_{J}(b)=o t(b)$ and $v_{J}(c)=o t(c)$ the result to be proved follows immediately from Lemma 4.5.

In the general case we reason as follows. We can assume $v_{J}(b)>1, v_{J}(c)>1$, as otherwise the result follows easily from the definitions. Thus, as before, there are $b^{\prime} \equiv b \bmod J+K, c^{\prime} \equiv c \bmod J+K$ such that $v_{J}(b)=\operatorname{ot}\left(b^{\prime}\right)=v_{J}\left(b^{\prime}\right), v_{J}(c)=$ ot $\left(c^{\prime}\right)=v_{J}\left(c^{\prime}\right)$. By the special case, $v_{J}\left(b^{\prime} c^{\prime}\right) \leq v_{J}\left(b^{\prime}\right) \odot v_{J}\left(c^{\prime}\right)$. It remains to show that $v_{J}(b c) \leq v_{J}\left(b^{\prime}\right) \odot v_{J}\left(c^{\prime}\right)$. We write $b=b^{\prime}+j+r$ and $c=c^{\prime}+j_{1}+r_{1}$, with $j, j_{1} \in J$ and $r, r_{1} \in K$. Then $b c \equiv b^{\prime} c^{\prime}+r c^{\prime}+r_{1} b^{\prime} \bmod J+K$. We can assume $v_{J}(b c)>1$, as otherwise there is nothing to prove. Thus $v_{J}(b c)=v_{J}\left(b^{\prime} c^{\prime}+r c^{\prime}+r_{1} b^{\prime}\right)$. By part $1, v_{J}(b c) \leq \max \left\{v_{J}\left(b^{\prime} c^{\prime}\right), v_{J}\left(c^{\prime}\right), v_{J}\left(b^{\prime}\right)\right\} \leq \max \left\{v_{J}\left(b^{\prime}\right) \odot v_{J}\left(c^{\prime}\right), v_{J}\left(c^{\prime}\right), v_{J}\left(b^{\prime}\right)\right\}=$ $v_{J}\left(b^{\prime}\right) \odot v_{J}\left(c^{\prime}\right)$.

\section{PRINCIPAL AND RESIDUAL ORDINAL-VALUES}

Definition 6.1. Given $b=\sum_{\beta} b_{\beta} t^{\beta} \in K((\mathbf{G}))$ and $\gamma \in \mathbf{G}$, we define:

1. $b_{\mid \gamma}=\sum_{\beta \leq \gamma} b_{\beta} t^{\beta}$.

2. $b^{\mid \gamma}=t^{-\gamma} b_{\mid \gamma}$.

We call $b_{\mid \gamma}$ the truncation of $b$ at $\gamma$ and we call the equivalence class of $b^{\mid \gamma}$ modulo $J$ the germ of $b$ at $\gamma$.

The supports of $b_{\mid \gamma}$ and $b^{\mid \gamma}$ differ only by a translation. The supremum of the support of $b_{\mid \gamma}$ is $\leq \gamma$. The supremum of the support of $b^{\mid \gamma}$ is $\leq 0$. So in particular $b^{\mid \gamma} \in K\left(\left(\mathbf{G}^{\leq 0}\right)\right)$.

Remark 6.2. $b^{\mid \gamma} \equiv c^{\mid \gamma} \bmod J$ iff for every $\delta$ sufficiently close to $\gamma$ the coefficients of $t^{\delta}$ in $b$ and $c$ coincide.

Remark 6.3. $b^{\mid \gamma} \notin J$ if and only if $\gamma$ is in the topological closure of the support of $b$ with respect to the order topology of $\mathbf{G}$. So in particular $b^{\mid \gamma} \notin J$ only for $\gamma$ ranging through a well ordered set.

The proof of the multiplicative property $v_{J}(b c)=v_{J}(b) \odot v_{J}(c)$ is simpler if $v_{J}(b)$ and $v_{J}(c)$ (or at least one of the two) are multiplicative principal ordinals. I recommend to the reader to make this simplifying assumption on a first reading. To deal with the general case we will define the "principal value" of a series $b$, in such a way that the principal value is always multiplicative principal and coincides with the ordinal value when the latter is multiplicative principal. Roughly speaking, the idea is that any series can be thought of as a series of multiplicative principal ordinal value, provided we allow the coefficients themselves to be series. For instance, a series of ordinal value $\omega^{3}$ (which is not multiplicative principal) can be also understood as a series of ordinal value $\omega$ (which is multiplicative principal) whose coefficients are series of ordinal value $\omega^{2}$. To be quite honest, this simple idea does not actually work so neatly, and it is somehow hidden in the technicalities that follow. However, as a motivation, it may be useful to keep it in mind.

Definition 6.4. Given $b \in K\left(\left(\mathbf{G}^{\leq 0}\right)\right)$ with $v_{J}(b)>1$, we know that $v_{J}(b)$ has the form $\omega^{\beta}$ for some ordinal $\beta>0$. From the Cantor normal form of $\beta$ it follows that $v_{J}(b)$ can be written uniquely as a product $\rho_{1} \rho_{2} \ldots \rho_{n}$, where $\rho_{1} \geq \rho_{2} \geq \ldots \geq \rho_{n}>$ 1 are multiplicative principal ordinals. We define:

1. $v_{J}^{p}(b)=\rho_{n}=$ the principal value of $b$, 
2. $v_{J}^{r}(b)=\rho_{1} \rho_{2} \ldots \rho_{n-1}=$ the residual value of $b$, with the convention that the residual value is 1 if $v_{J}(b) \in \mathbf{M P}$ (i.e. if $n=1$ ).

So if $v_{J}(b)=\omega^{3}$, then $v_{J}^{p}(b)=\omega$ and $v_{J}^{r}(b)=\omega^{2}$.

Remark 6.5. The product $\rho_{1} \rho_{2} \ldots \rho_{n}$ coincides with the natural product $\rho_{1} \odot \rho_{2} \odot$ $\ldots \odot \rho_{n}$. More generally if $\rho \in \mathbf{M P}, \alpha \in \mathbf{H}$ and $\rho \geq \alpha$, then $\rho \alpha=\rho \odot \alpha$.

For every $b \in K\left(\left(\mathbf{G}^{\leq 0}\right)\right)$ of value $>1$ we have the decomposition:

$$
v_{J}(b)=v_{J}^{r}(b) v_{J}^{p}(b)
$$

with $v_{J}^{r}(b) \in \mathbf{H}$ and $v_{J}^{p}(b) \in \mathbf{M P}$.

Definition 6.6. Given $b \in K((\mathbf{G} \leq 0))$ with $v_{J}(b)>1$, define

$$
X(b)=\left\{\gamma<0 \mid v_{J}\left(b^{\mid \gamma}\right)=v_{J}^{r}(b)\right\} .
$$

Informally we think of $b$ as a "hyper-series" of ordinal value $v_{J}^{p}(b)$ whose coefficients are series of ordinal value $v_{J}^{r}(b)$. The support of this hyper-series is $X(b)$. It may be challenging to find the correct algebraic framework for these hyper-series.

Note that if $v_{J}(b)$ is multiplicative principal, then $X(b)$ is the set of all $\gamma<0$ with $v_{J}\left(b^{\mid \gamma}\right)=1$, namely the set of all isolated points of the support of $b$.

Remark 6.7. If $\gamma \in X(b)$, then either $v_{J}\left(b^{\mid \gamma}\right)=1$ or $v_{J}^{p}\left(b^{\mid \gamma}\right)=\rho_{n-1} \geq v_{J}^{p}(b)$.

For the following result we specialize to the case $\mathbf{G}=(\mathbf{R},+, 0, \leq)$.

Lemma 6.8. Let $b \in K\left(\left(\mathbf{R}^{\leq 0}\right)\right)$. If $v_{J}(b)>1$, then every sufficiently small nonempty final segment of $X(b)$ has order type equal to $v_{J}^{p}(b)$ and supremum $=0$.

Proof. Let $\rho=v_{J}^{r}(b)$ and let $(-\varepsilon, 0) \cap S_{b}$ be a stable interval for $b$. We first prove that $\sup X(b)=0$. We have $v_{J}(b)=\rho v_{J}^{p}(b)=\sup _{\alpha<v_{J}^{p}(b)} \rho \alpha$. Using the completeness of $\mathbf{R}$, we consider for each $\alpha<v_{J}^{p}(b)$ the supremum $\gamma_{\alpha} \in \mathbf{R}$ of the first $\rho(\alpha+1)$ elements of $(-\varepsilon, 0) \cap S_{b}$. Since $\rho \in \mathbf{H}$ and $\rho(\alpha+1)=\rho \alpha+\rho$, the principal part (see Fact 3.3) of $\rho(\alpha+1)$ is $\rho$. Therefore every sufficiently small final segment of the support of $b^{\mid \gamma_{\alpha}}$ has order type $\geq \rho$ (it can be $\rho$ or $\rho+1$ ). It follows that $b^{\mid \gamma_{\alpha}}$ has ordinal value $\rho$, namely $\gamma_{\alpha} \in X(b)$. Since $\sup _{\alpha} \gamma_{\alpha}=0$ we have $\sup X(b)=0$.

This also shows that $(-\varepsilon, 0) \cap X(b)$ has limit order type $\lambda$. By replacing $\varepsilon$ with a smaller $\varepsilon^{\prime}$, we can also assume that $\lambda \in \mathbf{H}$. Since $\alpha \mapsto \gamma_{\alpha}$ is a strictly increasing map from $v_{J}^{p}(b)$ into $(-\varepsilon, 0) \cap X(b)$, we have $\lambda \geq v_{J}^{p}(b)$.

To finish the proof it suffices to show the opposite inequality. We write $(-\varepsilon, 0) \cap S_{b}$ as the union $(-\varepsilon, 0) \cap \bigcup_{\gamma \in X(b)} S_{b_{\mid \gamma}}$. Now $(-\varepsilon, 0) \cap S_{b}$ has order type $v_{J}(b)=\rho v_{J}^{p}(b)$, while by Lemma 4.7 the union has order type $\geq \rho \lambda$. Thus $v_{J}^{p}(b) \geq \lambda$.

Lemma 6.9. Let $b, c \in K\left(\left(\mathbf{R}^{\leq 0}\right)\right)$. If $\rho \in \mathbf{H}$ and $v_{J}\left(c^{\mid \gamma}\right) \geq \rho$ for every $\gamma \in X(b)$ sufficiently close to zero, then $v_{J}(c) \geq \rho v_{J}^{p}(b)$.

Proof. We can assume $v_{J}(b)>1$. By Lemma 6.8 the set $X(b)$ has a final segment of order type $v_{J}^{p}(b)$. Since the intersection of $(-\infty, 0)$ with the support of $c$ is the union of the supports of the various $c_{\mid \gamma}$, an application of Lemma 4.7 gives $\rho v_{J}^{p}(b)$ as a lower bound for the order type of every non-empty final segment of $S_{c} \cap(-\infty, 0)$, from which we deduce the same lower bound for $v_{J}(c)$. 


\section{Convolution product}

Given a subset $B$ of $\mathbf{G}$, we denote by $\bar{B}$ its closure with respect to the order topology of $\mathbf{G}$. For simplicity, in this section we specialize to the case $\mathbf{G}=(\mathbf{R},+, 0, \leq)$, although the arguments go through under more general hypothesis.

Lemma 7.1. If $B, C$ are well ordered subsets of $\mathbf{R}$, then $\overline{B+C}=\bar{B}+\bar{C}$.

Proof. The non-trivial inclusion is $\subseteq$. Let $\gamma$ be in the closure of $B+C$. Then there is a sequence $\beta_{n}+\xi_{n}$ converging to $\gamma$ with $\beta_{n} \in B, \xi_{n} \in C(n<\omega)$. Since $B, C$ are well-ordered, by taking a subsequence we can assume $\beta_{n} \leq \beta_{n+1}$ and $\xi_{n} \leq \xi_{n+1}$ for every $n$. This implies that the sequences $\left(\beta_{n}\right)$ and $\left(\xi_{n}\right)$ are bounded, and therefore there exist the suprema $\bar{\beta}=\sup _{n} \beta_{n} \in \bar{B}$ and $\bar{\xi}=\sup _{n} \xi_{n} \in \bar{C}$. Hence $\gamma=\bar{\beta}+\bar{\xi}$, and we are done.

Remark 7.2. The lemma does not hold without the assumption that $B, C$ are well ordered.

Remark 7.3. The lemma fails if $\mathbf{R}$ is replaced by the rationals $\mathbf{Q}$.

Given two well-ordered subsets $B, C$ of $\mathbf{R}$ and $\gamma \in \mathbf{R}$, it is easy to see that the intersection of the straight line $L_{\gamma}=\{(x, y) \mid x+y=\gamma\}$ with the set of points $B \times C \subseteq \mathbf{R}^{2}$ is finite. We have:

Lemma 7.4. Let $B, C$ be closed and well-ordered subsets of $\mathbf{R}$. Given $\gamma \in \mathbf{R}$, let $\delta \leq \gamma$ be sufficiently close to $\gamma$. Then for every point $\left(\beta^{\prime}, \xi^{\prime}\right) \in B \times C$ on the line $L_{\delta}$ there is exactly one point $(\beta, \xi) \in B \times C$ on the line $L_{\gamma}$ with $\beta^{\prime} \leq \beta, \xi^{\prime} \leq \xi$. (See Figure 1, where we have displayed the finitely many points of $B \times C$ which lie on the lines $L_{\gamma}$ and $L_{\delta}$.)

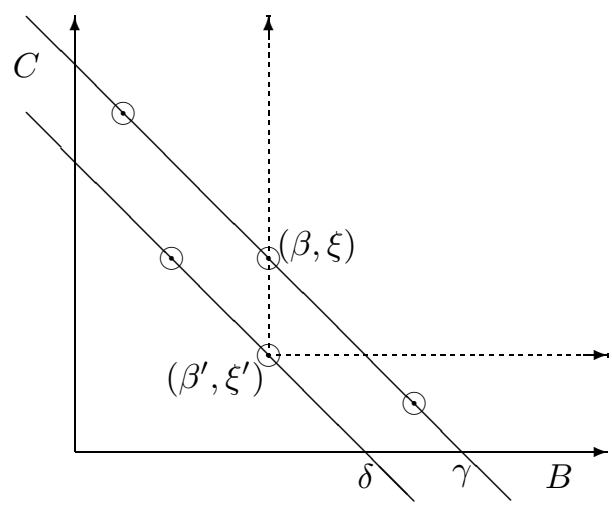

Figure 1

Proof. Uniqueness: As already remarked, $L_{\gamma}$ has only finitely many points in $B \times C$. If $\beta_{1}+\xi_{1}=\gamma, \ldots, \beta_{n}+\xi_{n}=\gamma$ are all the solutions of $x+y=\gamma$ in $B \times C$, then it suffices to take $\gamma-\delta$ smaller than the differences between any two $\beta_{i}$.

Existence: For a contradiction suppose that there is a sequence $\left(\gamma_{n} \mid n\right)$ converging to $\gamma$ from below and for each $n$ a point $\left(\beta_{n}, \xi_{n}\right) \in B \times C$ on the line $L_{\gamma_{n}}$ such that there is no point $(\beta, \xi) \in B \times C$ on $L_{\gamma}$ with $\beta_{n} \leq \beta, \xi_{n} \leq \xi$. Since $B, C$ are well ordered, by taking a subsequence we can assume that for each $n, \beta_{n} \leq \beta_{n+1}$ and 
$\xi_{n} \leq \xi_{n+1}$. Then the suprema $\beta=\sup _{n} \beta_{n}$ and $\xi=\sup _{n} \xi_{n}$ exist and $(\beta, \xi) \in B \times C$ (as $B, C$ are closed), a contradiction.

Lemma 7.5. Let $b, c \in K\left(\left(\mathbf{R}^{\leq 0}\right)\right)$ and $\gamma \in \mathbf{R} \leq 0$.

1. There are only finitely many pairs $(\beta, \xi) \in \mathbf{R} \leq 0 \times \mathbf{R} \leq 0$ with $\beta+\xi=\gamma$ and $b^{\mid \beta} c^{\mid \xi} \not \equiv 0 \bmod J$.

2. $(b c)^{\mid \gamma} \equiv \sum_{\beta+\xi=\gamma} b^{\mid \beta} c^{\mid \xi} \bmod J \quad$ (convolution formula).

Proof. 1. If $b^{\mid \beta} c^{\mid \xi} \not \equiv 0 \bmod J$, then $\beta$ belongs to the closure $\bar{B}$ of the support $B$ of $b$ and $\xi$ belongs to the closure $\bar{C}$ of the support $C$ of $c$. Point 1 follows from the fact that $\bar{B}$ and $\bar{C}$ are well ordered.

2. By Lemma 7.1 and Remark 6.3 we can assume $\gamma \in \bar{B}+\bar{C}$; otherwise both sides are $\equiv 0 \bmod J$. Let $[\phi]=0$ if $\phi=$ false, $[\phi]=1$ if $\phi=$ true. Lemma 7.4 can be rephrased as:

$$
\sum_{(\beta, \xi) \in \bar{B} \times \bar{C}}[\beta+\xi=\gamma]\left[\beta^{\prime} \leq \beta\right]\left[\xi^{\prime} \leq \xi\right]=1
$$

provided $\beta^{\prime}+\xi^{\prime}=\delta$ with $\delta \leq \gamma$ sufficiently close to $\gamma$. The convolution formula says that $\gamma$ is not in the closure of the support of

$$
d=(b c)_{\mid \gamma}-\sum_{(\beta, \xi) \in \bar{B} \times \bar{C}}[\beta+\xi=\gamma] b_{\mid \beta} c_{\mid \xi}
$$

So let us take $\delta \leq \gamma$ sufficiently close to $\gamma$ and let us show that the coefficient of $t^{\delta}$ in $d$ is zero. Let $b=\sum_{\beta} b_{\beta} t^{\beta}$ and $c=\sum_{\gamma} c_{\xi} t^{\xi}$.

The coefficient of $t^{\delta}$ in $(b c)_{\mid \gamma}$ is

$$
\sum_{\beta^{\prime}, \xi^{\prime}}\left[\beta^{\prime}+\xi^{\prime}=\delta\right] b_{\beta^{\prime}} c_{\xi^{\prime}}
$$

The coefficient of $t^{\delta}$ in $\sum_{(\beta, \xi) \in \bar{B} \times \bar{C}}[\beta+\xi=\gamma] b_{\mid \beta} c_{\mid \xi}$ is equal to

$$
\sum_{(\beta, \xi) \in \bar{B} \times \bar{C}}[\beta+\xi=\gamma]\left(\sum_{\beta^{\prime}, \xi^{\prime}}\left[\beta^{\prime}+\xi^{\prime}=\delta\right]\left[\beta^{\prime} \leq \beta\right]\left[\xi^{\prime} \leq \xi\right] b_{\beta^{\prime}} c_{\xi^{\prime}}\right)
$$

Taking the difference, we see that the coefficient of $t^{\delta}$ in $d$ is given by

$$
\sum_{\beta^{\prime}, \xi^{\prime}}\left[\beta^{\prime}+\xi^{\prime}=\delta\right]\left(1-\sum_{(\beta, \xi) \in \bar{B} \times \bar{C}}[\beta+\xi=\gamma]\left[\beta^{\prime} \leq \beta\right]\left[\xi^{\prime} \leq \xi\right]\right) b_{\beta^{\prime}} c_{\xi^{\prime}}
$$

and it is therefore equal to zero by equation (5).

Remark 7.6. By induction one can extend the lemma to more than two factors. For instance, $(a b c)^{\mid \gamma} \equiv \sum_{\alpha, \beta, \xi}[\alpha+\beta+\xi=\gamma] a^{\mid \alpha} b^{\mid \beta} c^{\mid \xi} \bmod J$

Lemma 7.7. Let $b, c \in K((\mathbf{R} \leq 0))$ be such that $v_{J}^{p}(b) \leq v_{J}^{p}(c)$. Let $\equiv$ be the congruence relation modulo $J$. Then for $\gamma \in \mathbf{R}^{<0}$ sufficiently close to zero:

$$
\begin{aligned}
(b c)^{\mid \gamma} \equiv & b^{\mid \gamma} c+b c^{\mid \gamma} \\
& + \text { terms of value }<v_{J}^{r}(b) \odot v_{J}(c)
\end{aligned}
$$

Proof. By the submultiplicative property it suffices to show that if $\beta, \xi \in \mathbf{R}^{<0}$ are sufficiently close to zero, then $v_{J}\left(b^{\mid \beta}\right) \odot v_{J}\left(c^{\mid \xi}\right)<v_{J}^{r}(b) \odot v_{J}(c)$. There are ordinals $\alpha_{1}<v_{J}^{p}(b), \alpha_{2}<v_{J}^{p}(c)$ such that $v_{J}\left(b^{\mid \beta}\right) \leq v_{J}^{r}(b) \alpha_{1}$ and $v_{J}\left(c^{\mid \xi}\right) \leq v_{J}^{r}(c) \alpha_{2}$. Since 
$v_{J}^{p}(c) \in \mathbf{M P}$, we have $\alpha_{1} \odot \alpha_{2}<v_{J}^{p}(c)$. Hence $v_{J}\left(b^{\mid \beta}\right) \odot v_{J}\left(c^{\mid \xi}\right) \leq v_{J}^{r}(b) \alpha_{1} \odot$ $v_{J}^{r}(c) \alpha_{2}<v_{J}^{r}(b) \odot v_{J}^{r}(c) \odot v_{J}^{p}(c)=v_{J}^{r}(b) \odot v_{J}(c)$.

\section{The MAIN LEMma}

Definition 8.1. For $\alpha \in O R$ and $k<\omega$, let $\bigodot^{k} \alpha=\alpha \odot \ldots \odot \alpha$ with $k$ occurrences of $\alpha$.

Lemma 8.2. Let $b, c \in K((\mathbf{R} \leq 0))$ be such that $v_{J}^{p}(b) \leq v_{J}^{p}(c)$ and let $k>0$. Suppose that for every $\gamma \in X(b)$ sufficiently close to zero we have

$$
v_{J}\left(b^{\mid \gamma} b^{k-1} c^{2}\right)=\bigodot^{k-1} v_{J}(b) \odot v_{J}\left(b^{\mid \gamma}\right) \odot v_{J}(c) \odot v_{J}(c)
$$

Then:

$$
v_{J}\left(b^{k} c\right)=\bigodot^{k} v_{J}(b) \odot v_{J}(c)
$$

Proof. We write $\equiv$ for congruence modulo the ideal $J$. Let $\gamma \in X(b)$ be sufficiently close to zero. Reasoning as in Lemma 7.7 we have

$$
\begin{aligned}
\left(b^{k} c\right)^{\mid \gamma} \equiv & k b^{k-1} b^{\mid \gamma} c+b^{k} c^{\mid \gamma} \\
& + \text { terms of value }<\bigodot \bigodot_{J}(b) \odot v_{J}^{r}(b) \odot v_{J}(c)
\end{aligned}
$$

The crucial idea is to multiply the above equation by $c$, obtaining

$$
\begin{aligned}
c\left(b^{k} c\right)^{\mid \gamma} \equiv & k b^{k-1} b^{\mid \gamma} c^{2}+b^{k} c c^{\mid \gamma} \\
& + \text { terms of value }<\bigodot v_{J}(b) \odot v_{J}^{r}(b) \odot v_{J}(c) \odot v_{J}(c)
\end{aligned}
$$

Suppose for a contradiction that $v_{J}\left(b^{k} c\right)<\bigodot^{k} v_{J}(b) \odot v_{J}(c)$. We claim that the terms of small value of the above equation include also $b^{k} c c^{l \gamma}$, namely:

$$
v_{J}\left(b^{k} c c^{\mid \gamma}\right)<\bigodot^{k-1} v_{J}(b) \odot v_{J}^{r}(b) \odot v_{J}(c) \odot v_{J}(c)
$$

To prove the claim we write $\bigodot^{k} v_{J}(b) \odot v_{J}(c)=\left[\odot^{k-1} v_{J}(b) \odot v_{J}^{r}(b) \odot v_{J}(c)\right] v_{J}^{p}(b)$ (using $v_{J}^{p}(b) \leq v_{J}^{p}(c)$ ). Since ordinal multiplication is continuous in the second argument (unlike the natural product) and since $v_{J}\left(b^{k} c\right)<\odot^{k} v_{J}(b) \odot v_{J}(c)$, there must be some $\alpha_{1}<v_{J}^{p}(b) \leq v_{J}^{p}(c)$ such that

$$
v_{J}\left(b^{k} c\right) \leq\left[\bigodot^{k-1} v_{J}(b) \odot v_{J}^{r}(b) \odot v_{J}(c)\right] \alpha_{1} .
$$

On the other hand, if $\gamma$ is sufficiently small, then $v_{J}\left(c^{\mid \gamma}\right) \leq v_{J}^{r}(c) \alpha_{2}$ for some $\alpha_{2}<v_{J}^{p}(c)$. Since $v_{J}^{p}(c) \in \mathbf{M P}, \alpha_{1} \odot \alpha_{2}<v_{J}^{p}(c)$. Thus we obtain

$$
\begin{aligned}
v_{J}\left(b^{k} c c^{\mid \gamma}\right) & \leq v_{J}\left(b^{k} c\right) \odot v_{J}\left(c^{\mid \gamma}\right) \\
& \leq\left[\bigodot v_{J}(b) \odot v_{J}^{r}(b) \odot v_{J}(c)\right] \alpha_{1} \odot v_{J}^{r}(c) \alpha_{2} \\
& \left.<\bigodot_{k-1}^{k-1} v_{J}(b) \odot v_{J}^{r}(b) \odot v_{J}(c)\right] \odot v_{J}^{r}(c) \odot v_{J}^{p}(c) \\
& =\bigodot^{k-1} v_{J}(b) \odot v_{J}^{r}(b) \odot v_{J}(c) \odot v_{J}(c)
\end{aligned}
$$


The claim is thus proved, and from equation (6) we obtain

$$
\begin{aligned}
c\left(b^{k} c\right)^{\mid \gamma} \equiv & k b^{k-1} b^{\mid \gamma} c^{2} \\
& + \text { terms of value }<\bigodot \bigodot_{J}^{k-1} v_{J}(b) \odot v_{J}^{r}(b) \odot v_{J}(c) \odot v_{J}(c)
\end{aligned}
$$

By our hypothesis $v_{J}\left(b^{\mid \gamma} b^{k-1} c^{2}\right)=\bigodot^{k-1} v_{J}(b) \odot v_{J}\left(b^{\mid \gamma}\right) \odot v_{J}(c) \odot v_{J}(c)$. Since $\gamma \in X(b), v_{J}\left(b^{\mid \gamma}\right)=v_{J}^{r}(b)$. It then follows that

$$
v_{J}\left(c\left(b^{k} c\right)^{\mid \gamma}\right)=\bigodot^{k-1} v_{J}(b) \odot v_{J}^{r}(b) \odot v_{J}(c) \odot v_{J}(c)
$$

By the submultiplicative property, we can now divide by $c$ and conclude that

$$
v_{J}\left(\left(b^{k} c\right)^{\mid \gamma}\right) \geq \bigodot^{k-1} v_{J}(b) \odot v_{J}^{r}(b) \odot v_{J}(c)
$$

Since the above inequality holds for every $\gamma \in X(b)$ sufficiently close to zero, we obtain by Lemma 6.9:

$$
\begin{aligned}
v_{J}\left(b^{k} c\right) & \geq \bigodot^{k-1} v_{J}(b) \odot v_{J}^{r}(b) \odot v_{J}(c) \odot v_{J}^{p}(b) \\
& =\bigodot^{k} v_{J}(b) \odot v_{J}(c)
\end{aligned}
$$

The opposite inequality also holds (by the submultiplicative property), and we are done.

\section{INDUCTION}

Definition 9.1. A formal expression is an element of the free commutative monoid generated by $\left\{x \in K\left(\left(\mathbf{R}^{\leq 0}\right)\right) \mid v_{J}(x)>1\right\}$.

Given $b_{0}, \ldots, b_{n} \in\left\{x \in K\left(\left(\mathbf{R}^{\leq 0}\right)\right) \mid v_{J}(x)>1\right\}$ and integers $k_{0}, \ldots, k_{n} \geq 1$, we may consider the product $b_{0}^{k_{0}} \cdot \ldots \cdot b_{n}^{k_{n}}$ either as an element of $K((\mathbf{R} \leq 0))$ or as a formal expression. We will introduce a complexity measure on formal expressions such that under the hypothesis of Lemma 8.2 plus some additional assumptions the complexity of $b^{\mid \gamma} b^{k-1} c^{2}$ is smaller than that of $b^{k} c$.

Definition 9.2. Given ordinals $\alpha_{0}, \ldots, \alpha_{m}$, let $\left[\alpha_{0}, \ldots, \alpha_{m}\right]=\omega^{\alpha_{0}} \oplus \ldots \oplus \omega^{\alpha_{m}}$. Note that $\left[\alpha_{0}, \ldots, \alpha_{m}\right]$ decreases if any $\alpha_{i}$ is replaced by any finite number of smaller ordinals.

Definition 9.3. Let $\prec$ be a fixed well ordering on $K\left(\left(\mathbf{R}^{\leq 0}\right)\right.$ ) (actually a linear ordering suffices). Given a formal expression $w=b_{0}^{k_{0}} \cdot \ldots \cdot b_{n}^{k_{n}}$ with all the $b_{j}$ 's distinct, let $Y$ be the set of all the elements of $\left\{b_{0}, \ldots, b_{n}\right\}$ of minimal principal value and let $Z \subseteq Y$ be the set of all the elements of $Y$ of maximal ordinal-value. If $b_{i}$ is the $\prec$-least element of $Z$, then $b_{i}$ will be called the selected factor of $w$ and the integer $k_{i}$ will be called the selected exponent. We then say that $b_{j}$ $(j \in\{0, \ldots, n\})$ is a relevant factor if its ordinal-value is bigger than or equal to the ordinal-value of the selected factor. The complexity of the formal expression $w$ is defined as the ordinal

$$
\omega\left[\alpha_{0}, \ldots, \alpha_{m}\right]+k
$$

where $k$ is the selected exponent and $\alpha_{0}, \ldots, \alpha_{m}(m \leq n)$ is the sequence of the ordinal-values of the relevant factors. 
Any formal expression can be reduced to a formal expression in which the $b_{j}$ 's are distinct by replacing $b^{k_{1}} b^{k_{2}}$ with $b^{k_{1}+k_{2}}$. The definition of complexity thus extends to all the formal expressions.

Remark 9.4. We have already noticed (Remark 6.7) that if $\gamma \in X(b)$, then either $v_{J}\left(b^{\mid \gamma}\right)=1$ or $v_{J}^{p}\left(b^{\mid \gamma}\right) \geq v_{J}^{p}(b)$. If $\gamma$ is sufficiently close to zero we also have $v_{J}\left(b^{l \gamma}\right)<v_{J}(b)$.

\section{Lemma 9.5. Let}

$$
w_{1}=b_{0}^{k_{0}} \cdot \ldots \cdot b_{n}^{k_{n}}
$$

be a formal expression. Suppose without loss of generality that $b_{0}$ is the selected factor and the elements $b_{i}$ are distinct. If $\gamma \in X\left(b_{0}\right)$ is sufficiently close to zero, then the complexity of $w_{1}$ is strictly greater than that of

$$
w_{2}=b_{0}^{\mid \gamma} b_{0}^{k_{0}-1} b_{1}^{2 k_{1}} \cdot \ldots \cdot b_{n}^{2 k_{n}}
$$

We agree that if $k_{0}-1=0$ we omit the factor $b_{0}^{k_{0}-1}$ and if $v_{J}\left(b_{0}^{\mid \gamma}\right)=1$ we omit $b_{0}^{\mid \gamma}$ (the possibility $v_{J}\left(b_{0}^{\mid \gamma}\right)=0$ is excluded since $\gamma \in X\left(b_{0}\right)$ ). Note that we are not claiming that the displayed factors of $w_{2}$ are all distinct.

Proof. Let $\omega\left[\alpha_{0}, \ldots, \alpha_{m}\right]+k$ be the complexity of $w_{1}$ (hence $k=k_{0}$ ).

Case 1. Suppose $k>1$. By the previous remark $b_{0}^{\mid \gamma}$ is neither the selected factor nor a relevant factor of $w_{2}$. Hence the selected factor of $w_{2}$ is $b_{0}$ and the complexity of $w_{2}$ is $\omega\left[\alpha_{0}, \ldots, \alpha_{m}\right]+(k-1)$.

Case 2. Suppose $k=1$. Then $w_{2}=b_{0}^{\mid \gamma} b_{1}^{2 k_{1}} \cdot \ldots \cdot b_{n}^{2 k_{n}}$. Now, regardless of which is the selected factor of $w_{2}$, the complexity of $w_{2}$ is of the form $\omega\left[\beta_{0}, \ldots, \beta_{r}\right]+p$, where $p \in \omega$ and $\left[\beta_{0}, \ldots, \beta_{r}\right]<\left[\alpha_{0}, \ldots, \alpha_{m}\right]$. Indeed, $\left[\beta_{0}, \ldots, \beta_{r}\right]$ is obtained from $\left[\alpha_{0}, \ldots, \alpha_{m}\right]$ by dropping $\alpha_{0}$ and replacing it by a finite number of ordinals strictly less than $\alpha_{0}$ (because $v_{J}\left(b_{0}^{\mid \gamma}\right)<v_{J}\left(b_{0}\right)$ and, for every $j \neq 0$, if $v_{J}\left(b_{j}\right) \geq v_{J}\left(b_{0}\right)$, then $v_{J}\left(b_{j}\right)$ was already present in the list $\left.\alpha_{0}, \ldots, \alpha_{m}\right)$. The desired result follows.

Lemma 9.6. Given elements $b_{0}, \ldots, b_{n} \in K\left(\left(\mathbf{R}^{\leq 0}\right)\right)$, we have:

$$
v_{J}\left(b_{0}^{k_{0}} \cdot \ldots \cdot b_{n}^{k_{n}}\right)=\bigodot^{k_{0}} v_{J}\left(b_{0}\right) \odot \ldots \odot \bigodot^{k_{n}} v_{J}\left(b_{n}\right) .
$$

Proof. We can assume $b_{0}, \ldots, b_{n}$ distinct, as otherwise we group together the equal factors $b_{i}$ by increasing the exponents. If some $b_{i}$ belongs to $J$ both sides are 0 . If some $b_{i}$ have value 1 we can delete it from both sides. So we can assume that all the elements $b_{i}$ have value $>1$. Without loss of generality $b_{0}$ is the selected factor. It then follows that for $\gamma \in X\left(b_{0}\right)$ sufficiently close to 0 , the complexity of $b_{0}^{\mid \gamma} b_{0}^{k_{0}-1} b_{1}^{2 k_{1}} \cdot \ldots \cdot b_{n}^{2 k_{n}}$ is strictly smaller than that of $b_{0}^{k_{0}} \cdot \ldots \cdot b_{n}^{k_{n}}$. By induction, $v_{J}\left(b_{0}^{\mid \gamma} b_{0}^{k_{0}-1} b_{1}^{2 k_{1}} \ldots b_{n}^{2 k_{n}}\right)=\bigodot^{k_{0}-1} v_{J}\left(b_{0}\right) \odot v_{J}\left(b_{0}^{\mid \gamma}\right) \odot \bigodot^{2 k_{1}} v_{J}\left(b_{1}\right) \odot \ldots \odot$ $\bigodot^{2 k_{n}} v_{J}\left(b_{n}\right)$. Let $c=b_{1}^{k_{1}} \cdot \ldots \cdot b_{n}^{k_{n}}$. Reasoning as in Case 2 of the proof of Lemma 9.5 , the complexity of $c$ is less than that of $b_{0}^{k_{0}} b_{1}^{k_{1}} \cdot \ldots \cdot b_{n}^{k_{n}}$. Hence by induction $v_{J}(c)=\bigodot^{k_{1}} v_{J}\left(b_{1}\right) \odot \ldots \odot \bigodot^{k_{n}} v_{J}\left(b_{n}\right)$. Putting everything together, we find that $v_{J}\left(b_{0}^{\mid \gamma} b_{0}^{k_{0}-1} c^{2}\right)=\bigodot^{k_{0}-1} v_{J}\left(b_{0}\right) \odot v_{J}\left(b_{0}^{\mid \gamma}\right) \odot v_{J}(c) \odot v_{J}(c)$. Thus by Lemma 8.2 $v_{J}\left(b_{0}^{k_{0}} c\right)=\bigodot^{k_{0}} v_{J}\left(b_{0}\right) \odot v_{J}(c)$, and we are done.

Theorem 9.7. For $b, c \in K\left(\left(\mathbf{R}^{\leq 0}\right)\right), v_{J}(b c)=v_{J}(b) \odot v_{J}(c)$.

Proof. Immediate from the previous lemma. 
Corollary 9.8. $J$ is a prime ideal of $K\left(\left(\mathbf{R}^{\leq 0}\right)\right)$.

Proof. $J$ is the set of all $x$ with $v_{J}(x)=0$. Now $v_{J}(x y)=0$ iff $v_{J}(x) \odot v_{J}(y)=0$ iff $v_{J}(x)=0$ or $v_{J}(y)=0$.

Corollary 9.9. Let $b, c \in K\left(\left(\mathbf{R}^{\leq 0}\right)\right)$. Then $o t(b c)=o t(b) \odot o t(c)$ provided there are ordinals $\beta$ and $\xi$ with $o t(b)=\omega^{\beta}$ and $o t(c)=\omega^{\xi}$.

Proof. After multiplying by suitable monomials we can reduce to the case $b, c \notin J$. Then $o t(b)=v_{J}(b)=\omega^{\beta}$ and $o t(c)=v_{J}(c)=\omega^{\xi}$. By the definition of $v_{J}$ and Remark 5.4, $\omega^{\beta \oplus \xi} \geq o t(b c) \geq v_{J}(b c)$. By Theorem $9.7 v_{J}(b c)=\omega^{\beta \oplus \xi}$, and we are done.

\section{IrReducible ELEMENTS of $K\left(\left(\mathbf{R}^{\leq 0}\right)\right)$}

Lemma 10.1. Given $0 \neq b \in K\left(\left(\mathbf{R}^{\leq 0}\right)\right)$, the set of ordinals $\left\{v_{J}\left(b^{\mid x}\right) \mid x \in \mathbf{R}^{\leq 0}\right\}$ has a maximum.

Proof. Let $\alpha$ be the supremum of $\left\{v_{J}\left(b^{\mid x}\right) \mid x \in \mathbf{R}^{\leq 0}\right\}$. If the supremum is not achieved, then $\alpha$ is a limit ordinal. Moreover $\alpha$ is countable because it is the supremum of a countable family of countable ordinals (any well-ordered subset of $\mathbf{R}$ is countable). Hence there exists an $\omega$-sequence $\alpha_{0}<\alpha_{1}<\alpha_{2}<\ldots$ such that $\alpha=\sup _{n} \alpha_{n}$. Let $x_{n}$ be such that $\alpha_{n}=v_{J}\left(b^{\mid x_{n}}\right)$. Since the $x_{n}$ range over a well-ordered set (the closure of the support of $b$ ), we can assume by choosing a subsequence that $x_{0} \leq x_{1} \leq x_{2} \leq \ldots$ Let $x=\sup _{n} x_{n}$. Every non-empty final segment of the support of $b^{\mid x}$ has order type $\geq \alpha_{n}$ for every $n$. Thus $v_{J}\left(b^{\mid x}\right)=\alpha$.

Definition 10.2. Given $0 \neq b \in K\left(\left(\mathbf{R}^{\leq 0}\right)\right)$, let $\alpha=\max \left\{v_{J}\left(b^{\mid x}\right) \mid x \in \mathbf{R}^{\leq 0}\right\}$. We say that $x \in \mathbf{R} \leq 0$ is the critical point of $b$ if $x$ is the smallest real number such that $v_{J}\left(b^{\mid x}\right)=\alpha$. This is well defined by the previous lemma and the fact that $\left\{y \mid v_{J}\left(b^{\mid y}\right) \neq 0\right\}$ is well ordered (is included in the closure of the support of $b$ ).

Remark 10.3. If $x$ is the critical point of $b, v_{J}\left(b^{\mid x}\right) \geq v_{J}(b)$.

Lemma 10.4. Let $b, c$ be non-zero elements of $K\left(\left(\mathbf{R}^{\leq 0}\right)\right)$ with critical points $x, y$ respectively. Then $v_{J}\left((b c)^{\mid x+y}\right)=v_{J}\left(b^{\mid x}\right) \odot v_{J}\left(c^{\mid y}\right)$.

Proof. We write $\equiv$ for congruence modulo $J$. By Theorem 9.7, by the choice of $x, y$ and by Lemma 7.5 we have $(b c)^{\mid x+y} \equiv b^{\mid x} c^{\mid y}+$ terms of value $<v_{J}\left(b^{\mid x}\right) \odot v_{J}\left(c^{\mid y}\right)$. The desired result follows.

Theorem 10.5. Suppose that $a \in K\left(\left(\mathbf{R}^{\leq 0}\right)\right)$ is not divisible by any monomial $t^{\gamma}$ with $\gamma<0$. If the order type of the support of a is either $\omega$ or of the form $\omega^{\omega^{\beta}}$, then both $a$ and $a+1$ are irreducible in $K\left(\left(\mathbf{R}^{\leq 0}\right)\right)$.

Proof. The hypothesis implies $1<\operatorname{ot}(a)=v_{J}(a)=v_{J}(a+1) \in \mathbf{M P}$. We show that $a$ is irreducible. The argument for $a+1$ is the same. Suppose $a=b c$ with $b, c$ not invertible in $K\left(\left(\mathbf{R}^{\leq 0}\right)\right)$. Then $v_{J}(a)=v_{J}(b) \odot v_{J}(c)$. Since $v_{J}(a) \in \mathbf{M P}$, either $v_{J}(b)$ or $v_{J}(c)$ must be 1 . Suppose $v_{J}(b)=1$. Then $v_{J}(c)=v_{J}(a)=\rho \in$ MP. Let $x, y \in \mathbf{R} \leq 0$ be the critical points of $b, c$ respectively. Then $v_{J}\left(a^{\mid x+y}\right)=$ $v_{J}\left(b^{\mid x}\right) \odot v_{J}\left(c^{\mid y}\right) \geq v_{J}(b) \odot v_{J}(c)=v_{J}(a)$. Since $v_{J}(a)=\operatorname{ot}(a)$, for every $u<0$ we have $v_{J}\left(a^{\mid u}\right)<v_{J}(a)$. Hence $x+y=0$, and therefore $x=y=0$. On the other hand, since $v_{J}(b)=1$, the support of $b$ contains 0 as an isolated point. The support of $b$ must contain some other points, because $b$ is not a unit. So $x<0$, a contradiction. 
As already remarked in the introduction, $\mathbf{R}$ can be replaced by $\mathbf{G}$ provided $\mathbf{G}$ is archimedean (since then $\mathbf{G}$ embeds in $\mathbf{R}$ ).

\section{Series With Finite SUPport}

Definition 11.1. $K[\mathbf{G}]$, the ring of $\mathbf{G}$-polynomials, is defined as the subring of $K((\mathbf{G}))$ consisting of all the series with finite support. We then define $K\left[\mathbf{G}^{\leq 0}\right]$ as $K[\mathbf{G}] \cap K((\mathbf{G} \leq 0))$.

When $\mathbf{G}=\mathbf{R}$ we can prove:

Theorem 11.2. Let $a$ be an irreducible element of $K\left[\mathbf{R}^{\leq 0}\right]$. Then a is irreducible in $K\left(\left(\mathbf{R}^{\leq 0}\right)\right)$.

Proof. Suppose $a=b c$ is a non-trivial factorization in $K\left(\left(\mathbf{R}^{\leq 0}\right)\right)$. Since $a$ has finite support, $v_{J}(a) \leq 1$. Moreover, since $a$ is irreducible in $K[\mathbf{R} \leq 0]$ we must have $v_{J}(a)=1$; otherwise $a$ would be divisible by some monomial $t^{\gamma}$ with $\gamma<0$. Thus $v_{J}(a)=v_{J}(b) \odot v_{J}(c)=1$, and therefore $v_{J}(b)=v_{J}(c)=1$. Let $x, y$ be the critical points of $b, c$ respectively. Suppose for a contradiction that $b \notin K\left[\mathbf{R}^{\leq 0}\right]$. Then $v_{J}\left(b^{\mid x}\right)>1$ and $x<0$. Hence $v_{J}\left(a^{\mid x+y}\right)=v_{J}\left(b^{\mid x}\right) \odot v_{J}\left(c^{\mid y}\right)>1$, contradicting the fact that $a$ has finite support.

By Biljacović's work this implies that $t^{-\sqrt{2}}+t^{-1}+1$ is irreducible in $K((\mathbf{R} \leq 0))$, as well as any other series with finite support whose exponents are not linearly dependent over $\mathbf{Q}$.

\section{Appendix: Reduction to the Case $\mathbf{G}=\mathbf{R}$ (BASED ON IDEAS OF GONSHOR AND MOURGUes)}

We prove the existence of irreducible elements in the ring $K((\mathbf{G} \leq 0))$, where $K$ is any field of characteristic zero (not even assumed to be orderable) and $\mathbf{G}=$ $(\mathbf{G},+, 0, \leq)$ is any abelian divisible ordered group. In the special case when $\mathbf{G}=\mathbf{R}$ (or more generally $\mathbf{G}$ is archimedean) this follows from Theorem 10.5.

In his book Gonshor sketches an argument to reduce the general case to the case $\mathbf{G}=\mathbf{R}$. He first proves that in order for a series to be irreducible, the elements of its support - excluding zero - must belong to the same archimedean class, i.e. given two non-zero elements of the support, say $\beta$ and $\xi$, there is a natural number $n$ with $n|\beta|>|\xi|$ and $n|\xi|>|\beta|$. He then argues from this fact that the interesting case is $\mathbf{G}=\mathbf{R}$. At the meeting "Model theory of fields" (Durham, July 22-Aug. 1, 1996) I gave a talk about the special case $\mathbf{G}=\mathbf{R}$, and I had the opportunity to speak with M.-H. Mourgues, who explained me how to turn Gonshor's idea into a complete proof using the fact that every element of $\mathbf{G}$ lies in the set-theoretic difference of two convex subgroups $\mu \subset$ Fin of $\mathbf{G}$ with archimedean quotient Fin $/ \mu$. The argument which follows is a result of that conversation.

Theorem 12.1. 1. The series $\sum_{n} t^{-1 / n}+1$ is irreducible in $K((\mathbf{G} \leq 0))$.

2. More generally, let $Q$ be an archimedean subgroup of $\mathbf{G}$ and suppose that $a \in K\left(\left(Q^{<0}\right)\right) \subset K\left(\left(\mathbf{G}^{\leq 0}\right)\right)$ has support of order type $\omega$ or $\omega^{\omega^{\alpha}}$ ( $\alpha$ an ordinal) and $a$ is not divisible by any monomial $t^{\gamma}$ with $\gamma \in Q^{<0}$. Then $a+1$ is irreducible in $K\left(\left(\mathbf{G}^{\leq 0}\right)\right)$.

Proof. We know by Theorem 10.5 that $a$ and $a+1$ are irreducible in $K\left(\left(Q^{\leq 0}\right)\right)$. Fix a positive element of $Q$ and call it 1. Let Fin $\subseteq \mathbf{G}$ be the intersection of all convex 
subgroups of $\mathbf{G}$ containing 1 (hence containing $Q$ ). We will be done if we show that $a+1$ is irreducible in $K\left(\left(F_{i n} \leq 0\right)\right)$. In fact if we have a non-trivial factorization $a+1=b c$ in $K(\mathbf{G} \leq 0)$, then $v(a+1)=v(b)+v(c)$, hence $v(a+1)<v(b)<0$ and $v(a+1)<v(c)<0$ ( $v$ is the natural valuation into $\mathbf{G}$, not the ordinal value). So by convexity of Fin the supports of $b, c$ are included in Fin $\leq 0$.

The problem has been reduced to showing that $a+1$ is irreducible in $K\left(\left(F_{i n} \leq 0\right)\right)$. Let $\mu \subseteq$ Fin be the union of all the convex subgroups of $\mathbf{G}$ not containing 1 (hence $Q \cap \mu=\{0\})$. Then $Q$ is contained in the set-theoretic difference Fin $\backslash \mu$. The quotient $\mathrm{Fin} / \mu$ is archimedean, because it contains no non-trivial convex subgroups. Since $\mu$ is convex Fin $/ \mu$ has a natural induced order. In a divisible abelian group every subgroup is a direct factor. Thus we have:

$$
\text { Fin }=H \oplus \mu
$$

where $H \simeq \mathrm{Fin} / \mu$ is archimedean and it can be chosen so that $Q \subseteq H$ because $Q \cap \mu=\{0\}$ and $\mathbf{G}$ is divisible. (See [Baer 40] or [Fuchs 70, vol. 1, Theorem 21.2].) The ordering on $H \oplus \mu$ (induced by $\mathbf{G}$ ) is lexicographic: $h+m<h^{\prime}+m^{\prime}$ if and only if either $h<h^{\prime}$ in $H$ or $h=h^{\prime}$ and $m<m^{\prime}$ in $\mu$. It follows that the non-positive elements of Fin are given by:

$$
\text { Fin }^{\leq 0}=\left(H^{<0} \oplus \mu\right) \cup \mu^{\leq 0}
$$

This induces a canonical identification

$$
\begin{aligned}
K\left(\left(\text { Fin }^{\leq 0}\right)\right) & =K((\mu))\left(\left(H^{<0}\right)\right) \oplus K\left(\left(\mu^{\leq 0}\right)\right) \\
& \subset K((\mu))\left(\left(H^{\leq 0}\right)\right)
\end{aligned}
$$

Indeed, a series $\sum_{\beta} b_{\beta} t^{\beta} \in K\left(\left(\right.\right.$ Fin $\left.\left.^{\leq 0}\right)\right)$ can be also thought of as a series $\sum_{\xi \in H} c_{\xi} t^{\xi}$ where $c_{\xi} \in K((\mu))$ is given by $\sum_{\beta \in M(\xi)} b_{\beta} t^{\beta-\xi}$ with $M(\xi):=\{\beta \mid \beta-\xi \in$ $\mu\}$.

Since $\mu$ is a subgroup of $\mathbf{G}, F:=K((\mu))$ is a field and we have

$$
K\left(\left(Q^{\leq 0}\right)\right) \subseteq F\left(\left(H^{\leq 0}\right)\right)
$$

By hypothesis $a \in K\left(\left(Q^{\leq 0}\right)\right)$ has order type $\omega$ or $\omega^{\omega^{\alpha}}$ and $a$ is not divisible by any monomial $t^{\gamma}$ with $\gamma<0$. This remains true even if we allow $\gamma$ to range over $H$, since $H$ is archimedean. So both assumptions on $a$ remain true if we see $a$ as an element of $F((H \leq 0)$ ). By Theorem 10.5 (and the archimedean property of $H$ ) $a$ and $a+1$ are irreducible in the ring $F\left(\left(H^{\leq 0}\right)\right)=K((\mu))\left(\left(H^{\leq 0}\right)\right)$.

It does not yet follow that $a+1$ is irreducible in the smaller ring $K\left(\left(\right.\right.$ Fin $\left.\left.^{\leq 0}\right)\right)=$ $K((\mu))\left(\left(H^{<0}\right)\right) \oplus K\left(\left(\mu^{\leq 0}\right)\right)$, because the units of the two rings are different: the units of $F\left(\left(H^{\leq 0}\right)\right)$ are the elements of $F=K((\mu))$, while those of $K\left(\left(F_{i n} \leq 0\right)\right)$ are the elements of $K$. What we can conclude however is that if $a+1=b c$ is a non-trivial factorization in $K\left(\left(F_{i n} \leq 0\right)\right.$ ) (where "non-trivial" means $\left.b, c \notin K\right)$, then either $b$ or $c$ is a unit of $F\left(\left(H^{\leq 0}\right)\right)$, namely it belongs to $F=K((\mu))$. Assume, without loss of generality, that $b \in K((\mu))$. Then actually $b \in K\left(\left(\mu^{\leq 0}\right)\right)$. We have:

$$
(a+1) b^{-1}=a b^{-1}+b^{-1}=c \in K\left(\left(\text { Fin }^{\leq 0}\right)\right)
$$

Since $K((\mu))$ is a field, $b^{-1} \in K((\mu))$. Since the units of $K((\mu))$ are the elements of $K$ and $b \in K\left(\left(\mu^{\leq 0}\right)\right)$ was assumed not in $K$, it follows that the support of $b^{-1}$ contains some strictly positive element. We reach a contradiction by showing that also the support of $a b^{-1}+b^{-1}$ contains some strictly positive element. To see this it suffices to observe that the support of $a b^{-1}$ is disjoint from that of $b^{-1}$, 
since the former is contained in $Q^{<0}+\mu$ while the latter is contained in $\mu$ (and $Q \cap \mu=\{0\})$.

Remark 12.2. In the above theorem $\mathbf{G}$ can be allowed to be a proper class. So Conway's series $\sum_{n} t^{-1 / n}+1$ is irreducible even in the ring of omnific integers.

\section{CONCluding REMARKS}

- We left open the question of whether the irreducible elements of $K\left(\left(\mathbf{G}^{\leq 0}\right)\right)$ generate prime ideals.

- Conway's book asks whether in $K\left(\left(\mathbf{G}^{\leq 0}\right)\right)$ (actually in the ring of omnific integers) any two factorizations have a common refinement. This would guarantee that factorizations are unique when they exist.

- A related question, which arose during a conversation with Franz-Victor and Salma Kuhlmann, is whether $K\left(\left(\mathbf{G}^{\leq 0}\right)\right) / J$ is a unique factorization domain. The existence of the ordinal value map $v_{J}$ immediately ensures that there are no infinite ascending chains of principal ideals, so every element is a product of irreducible elements.

- Although for the sake of proving the existence of irreducible elements one can reduce to the case $\mathbf{G}=\mathbf{R}$, it is still of interest to determine whether the ordinal-value map $v_{J}: K\left(\left(\mathbf{G}^{\leq 0}\right)\right) \rightarrow(O R, \odot)$ has the multiplicative property even in the case when $\mathbf{G}$ is an arbitrary ordered abelian divisible group. This would imply in particular that the ideal $J \subseteq K\left(\left(\mathbf{G}^{\leq 0}\right)\right)$ is prime. In our proofs the assumption $\mathbf{G}=\mathbf{R}$ was only used in two places: in the results of section 7-namely the proof of the "convolution formula" - and in Lemma 6.8.

- It may be of interest to develop a general theory of "surreal valuations" (valuations inside the field of surreal numbers).

- Does every exponential integer part (in the sense of Ressayre) of a real closed exponential field contain unboundedly many irreducible elements? A positive answer would give a completely new proof of the infinity of primes even for the standard integers. For a positive answer one could try to show that our criterion for irreducibility necessarily applies in the presence of exponentiation. For a negative answer one should find an analogue of the fact that the real closed field of the Puiseux series with real coefficients has an integer part without infinite irreducible elements. A general discussion of real closed exponential fields can be found in the papers of Ressayre and in the papers of S. Kuhlmann and F.-V. Kuhlmann cited in the bibliography.

- In connection with the previous question it would be interesting to axiomatize - as already observed by Ressayre - the class of all exponential integer parts of real closed fields. One can certainly add to the axioms of open induction some obvious axioms for exponentiation, but it is unclear whether further axioms are needed.

- Which are the diophantine equations solvable in the ring of omnific interger? And those solvable in the smallest ordered ring containing $(O R, \oplus, \odot, \leq)$ ?

- What is the first order theory of $K\left(\left(\mathbf{G}^{\leq 0}\right)\right)$ ? Does it change with $\mathbf{G}$ ? If so, then what is the theory of the whole class of such structures? Does it have the joint embedding property? (By the work of [Otero 93-2] the theory open induction plus the normality axiom scheme does.)

- Besides $K\left(\left(\mathbf{G}^{\leq 0}\right)\right)$, it may be interesting to study the $\operatorname{ring} K\left(\left(\mathbf{G}^{\geq 0}\right)\right)$. 
- Up to what extent does the ordinal value depend on the power series representation, rather than on the ring structure of $K\left(\left(\mathbf{G}^{\leq 0}\right)\right)$ ? Can we extend the notion of ordinal value to more general rings?

\section{ACKNOWLEDGMENTS}

I thank Angus Macintyre and Dave Marker for having called to my attention, in the summer of 1995, the problem of the existence of irreducible generalized power series, and for their encouragement on this project. I owe Dikran Dikranjan a great debt of gratitude for having helped me in the early phases of this work. At the beginning I was focusing on a particular question: whether the series $\sum_{n} t^{-1 / n}$ is irreducible in $\mathbf{R}\left(\left(\mathbf{R}^{\leq 0}\right)\right)$. We made several attempts to prove that this and other series of order type $\omega$ were irreducible, obtaining partial results by purely combinatorial methods which did not use the valuation-theoretic approach developed in this paper (we could for instance exclude a factorization with two factors, one of order type $\leq \omega$ and the other of order type $\left.\leq \omega^{n}\right)$. I warmly thank Marie-Helene Mourgues for her suggestions on how to deal with a non-archimedean group $\mathbf{G}$, and Jean Pierre Ressayre for his interest in my work and for inviting me for a short stay at the University of Paris VII. A preliminary version of this paper has been improved thanks to the valuable comments of Lou van den Dries, Daniel Pitteloud, Vincenzo Scianni, and the anonymous referees. I thank Fabrizio Broglia both for mathematical discussions and for his patient help with the local computing facilities.

\section{REFERENCES}

[Baer 40] R. Baer, Abelian groups that are direct summands of every containing abelian group, Bulletin of the American Mathematical Society 46 (1940), 800-806. MR 2:126i

[Berarducci - Otero 96] A. Berarducci, M. Otero, A recursive nonstandard model of normal open induction, Journal of Symbolic Logic 61, n. 4 (1996) 1228 - 1241. MR 98i:03049

[Biljacovic 96] D. Biljaković, Recursive models of open induction with infinite primes, Preprint, 1996.

[Boughattas 93] S. Boughattas, Résultats optimaux sur l'existence d'une partie entière dans les corps ordonnés, Journal of Symbolic Logic 58, n. 1 (1993) 326 - 333. MR 94h:03066

[Conway 76] J. H. Conway, On Numbers and Games, Academic Press, London 1976. MR 56:8365

[Dries et al.] L. van den Dries, A. Macintyre, D. Marker, Logarithmic-Exponential Power Series, Journal of the London Mathematical Society, ser. 2, 56 (1997) 417-434. CMP 98:09

[Dries et al. 94] L. van den Dries, A. Macintyre, D. Marker, The elementary theory of restricted analytic fields with exponentiation, Annals of Mathematics 140 (1994) 183-205. MR 95k:12015

[Dries 80-1] L. van den Dries, Which curves over $\boldsymbol{Z}$ have points with coordinates in a discrete ordered ring?, Transactions of the American Mathematical Society 264 (1981) 181 - 189. MR 82i:03046

[Dries 80-2] L. van den Dries, Some model theory and number theory for models of weak systems of arithmetic, in: "Model Theory of Algebra and Arithmetic" (Karpacz, 1979; L.Pacholski et al., eds.), Lecture Notes in Mathematics 834, Springer-Verlag, Berlin-Heidelberg 1980, 346 - 362. MR 82f:03029

[Endler 72] O. Endler, Valuation Theory, Springer-Verlag, Berlin-Heidelberg 1972. MR 50:9847

[Fuchs 63] L. Fuchs, Partially ordered algebraic systems, Pergamon Press, Oxford 1963. MR 30:2090

[Fuchs 70] L. Fuchs, Infinite Abelian groups (2 volumes), Academic Press, New York-London, 1970, 1973. MR 41:333; MR 50:2362

[Gleyzal 37] A. Gleyzal, Transfinite numbers, Proceedings of the National Academy of Sciences 23 (1937) $581-587$.

[Gonshor 86] H. Gonshor, An introduction to the theory of surreal numbers, Cambridge University Press, Cambridge 1986. MR 88b:04002 
[Hahn 07] H. Hahn, Über die nichtarchimedischen Grössensysteme, Sitzungsberichte der Kaiserlichen Akademie der Wissenschaften, Wien, section IIa, 116 (1907) 601 - 655.

[Hausdorff 27] F. Hausdorff, Mengenlehre, Berlin, 1927.

[Kaplansky 42] I. Kaplansky, Maximal fields with valuations, Duke Mathematical Journal 9 (1942) 303 - 321. MR 3:264a

[Krull 32] W. Krull, Algemeine Bewertungstheorie, Journal für die reine und angewandte Mathematik 167 (1932) 160 - 196.

[Kuhlmann-Kuhlmann 94] F.-V. Kuhlmann, S. Kuhlmann, On the structure of nonarchimedean exponential field II, Communications in Algebra 22, n. 12 (1994) 5079 - 5103. MR 96h:12009

[Kuhlmann 95] S. Kuhlmann, On the structure of nonarchimedean exponential field I, Archive of Mathematical Logic 34 (1995) 145 - 182. MR 96g:12008

[Kuhlmann et al. 96] F.-V. Kuhlmann, S. Kuhlmann, S. Shelah, Exponentiation in power series fields, Proceedings of the American Mathematical Society 125 (1997) 3177-3183. MR 97m:12007

[Macintyre-Marker 89] A. Macintyre, D. Marker, Primes and their residues rings in models of open induction, Annals of Pure and Applied Logic 43 (1989) 57 - 77. MR 90g:03042

[MacLane 39] S. MacLane, The universality of formal power series fields, Bulletin of the American Mathematical Society 45 (1939) 888 - 890. MR 1:102c

[Moniri 94] M. Moniri, Recursive models of open induction of prescribed finite transcendence degree $>1$ with cofinal twin primes, C.R. Acad. Sci. Paris, Ser. I, Math. 319 (1994) 903-908. MR 96b:03046

[Mourgues 93] M.-H. Mourgues, Applications des corps de séries formelles à l'étude des corps réel clos et des corps exponentiels, Thèse de doctorat, Univeristé de Paris 7, Paris 1993.

[Mourgues-Ressayre 93] M. H. Mourgues, J.-P. Ressayre, Every real closed field has an integer part, Journal of Symbolic Logic (1993) 641 - 647. MR 95e:03168

[Neumann 49] B. H. Neumann, On ordered division rings, Transactions of the American Mathematical Society 66 (1949) $202-252$. MR 11:311f

[Otero 90] M. Otero, On diophantine equations solvable in models of open induction, Journal of Symbolic Logic 55 (1990) 779 - 786. MR 91i:03107

[Otero 93-1] M. Otero, Quadratic forms in normal open induction, Journal of Symbolic Logic 58, n. 2 (1993) 456 - 476. MR 94h:03068

[Otero 93-2] M. Otero, The joint embedding property in normal open induction, Annals of Pure and Applied Logic 60 (1993) 275 - 290. MR 94e:03037a

[Pohlers 80] W. Pohlers, Proof Theory, Lecture Notes in Mathematics 1407 (A. Dold, B. Eckmann, F. Takens, eds.), Springer-Verlag, Berlin Heidelberg 1989. MR 91h:03078

[Ressayre 93] J. P. Ressayre, Integer parts of real closed exponential fields, in: "Arithmetic, Proof theory and Computational Complexity" (P. Clote and J. Krajíček eds.), Oxford University Press, Oxford 1993, 278-288. MR 94e:03001

[Ressayre 95] J. P. Ressayre, Survey on transfinite series and their applications, Manuscript 1995.

[Ribenboim 68] P. Ribenboim, Théorie des valuation, Les presses de l'université de Montréal, Montréal 1968. MR 40:2670

[Ribenboim 92] P. Ribenboim, Fields: Algebrically closed and others, Manuscripta Mathematica 75 (1992) 115 - 166. MR 93f: 13014

[Shepherdson 64] J. Shepherdson, A nonstandard model for a free variable fragment of number theory, Bulletin de l'Académie Polonaise des Sciences 12 (1964) 79 - 86. MR 28:5002

[Sikorski 48] R. Sikorski, On an ordered algebraic field, Comptes-Rendues de l'Academie des Sciences et Lettres de Varsovie. 41 (1948) 61-96. MR 12:667f

[Wilkie 78] A. Wilkie, Some results and problems on weak systems of arithmetic, in: "Logic Colloquium'77" (A. Macintyre et al., eds.), North-Holland, Amsterdam 1978, 285 - 296. MR 81c:03050

Università di Pisa, Dipartimento di Matematica, Via Buonarroti 2, 56127 Pisa, Italy E-mail address: berardu@dm.unipi.it 